\title{
Steps toward determination of the size and structure of the broad-line region in active nuclei. 7: Variability of the optical spectrum of NGC 5548 over years
}

\section{Citation}

Peterson, B. M., P. Berlind, R. Bertram, N. G. Bochkarev, D. Bond, M. S. Brotherton, J. R. Busler, et al. 1994. "Steps Toward Determination of the Size and Structure of the Broad-Line Region in Active Nuclei. 7: Variability of the Optical Spectrum of NGC 5548 over Years." The Astrophysical Journal 425 (April): 622. doi:10.1086/174009.

\section{Published Version}

10.1086/174009

\section{Permanent link}

http://nrs.harvard.edu/urn-3:HUL.InstRepos:32955265

\section{Terms of Use}

This article was downloaded from Harvard University's DASH repository, and is made available under the terms and conditions applicable to Other Posted Material, as set forth at http:// nrs.harvard.edu/urn-3:HUL.InstRepos:dash.current.terms-of-use\#LAA

\section{Share Your Story}

The Harvard community has made this article openly available.

Please share how this access benefits you. Submit a story. 


\title{
STEPS TOWARD DETERMINATION OF THE SIZE AND STRUCTURE OF THE BROAD-LINE REGION IN ACTIVE GALACTIC NUCLEI. VII. VARIABILITY OF THE OPTICAL SPECTRUM OF NGC 5548 OVER 4 YEARS
}

\author{
B. M. Peterson, ${ }^{1}$ P. Berlind,${ }^{2}$ R. Bertram,${ }^{1,3}$ N. G. BochKarev, ${ }^{4}$ D. Bond,${ }^{5}$ M. S. Brotherton, ${ }^{6}$ \\ J. R. Busler, ${ }^{7}$ K. K. Chuvaev, ${ }^{8}$ R. D. Cohen, ${ }^{9}$ M. Dietrich, ${ }^{10}$ M. Elvis, ${ }^{2}$ A. V. Filippenko, ${ }^{11}$ \\ C. B. Foltz, ${ }^{12}$ P. M. Garnavich, ${ }^{5}$ L. C. Ho, ${ }^{11}$ E. Horine, ${ }^{2,13}$ K. Horne, ${ }^{14,15}$ J. P. Huchra, ${ }^{2}$ \\ W. Kollatschny, ${ }^{10}$ K. T. Korista, ${ }^{1,14,16}$ M. A. Malkan, ${ }^{17}$ T. Matheson, ${ }^{11}$ M. Mignoli, ${ }^{18}$ \\ S. L. Morris, ${ }^{5,19}$ L. Nazarova, ${ }^{20}$ J. Penfold, ${ }^{7,21}$ J. Peters, ${ }^{2}$ R. W. Pogge, ${ }^{1}$ V. I. Pronik, \\ B. Rush, ${ }^{17}$ S. G. Sergeev, ${ }^{8}$ A. I. Shapovalova, ${ }^{22}$ J. C. Shields, ${ }^{1,11,23}$ G. M. Stirpe, ${ }^{18}$ \\ S. ToKarz, ${ }^{2}$ R. M. WAGNer, ${ }^{1,3}$ R. J. WeYMANN, ${ }^{16}$ R. J. White, ${ }^{1,17}$ B. J. Wilkes, ${ }^{2}$ \\ D. Wills, ${ }^{6}$ B. J. Wills, ${ }^{6}$ C. Winge, ${ }^{1,24}$ AND P. F. Younger ${ }^{5}$ \\ Received 1993 July 23; accepted 1993 October 25
}

\begin{abstract}
We report on the results of a continuation of a large monitoring program of optical spectroscopy of the Seyfert 1 galaxy NGC 5548. The new observations presented here were obtained between 1990 December and 1992 October, and extend the existing database to nearly 1400 days, dating back to 1988 December. The continuum variations are generally smooth and well-resolved, except during the third year of this 4 year project, when the variations were apparently more rapid and of lower amplitude than observed at other times. The broad $\mathrm{H} \beta$ emission line is found to vary in response to the continuum variations with a lag of about 18 days, but with some changes from year to year. The $\mathrm{H} \beta$ transfer functions for each of the 4 yr and for the entire 4 yr database are derived by using a maximum entropy method.
\end{abstract}

Subject headings: galaxies: active - galaxies: individual (NGC 5548) - galaxies: nuclei — galaxies: Seyfert

\section{INTRODUCTION}

Intensive spectroscopic monitoring programs are now providing a wealth of detail about the structure and size of the broad-line region (BLR) in active galactic nuclei (AGNs) and on the nature of the continuum and its relationship to the emission lines (see Peterson 1993 for a review). The bright Seyfert 1 galaxy NGC 5548 is one of the sources that has received considerable attention in these efforts. Beginning in late 1988, the ultraviolet spectrum of this source was observed once every 4 days for an 8 month period with the International Ultraviolet Explorer (Clavel et al. 1991; hereafter Paper I). A concurrent ground-based campaign to monitor the variability of the optical spectrum was also carried out (Peterson et al.

\footnotetext{
${ }^{1}$ Department of Astronomy, Ohio State University, 174 West 18th Avenue, Columbus, OH 43210.

${ }^{2}$ Harvard-Smithsonian Center for Astrophysics, 60 Garden Street, Cambridge, MA 02138 .

${ }^{3}$ Mailing address: Lowell Observatory, Mars Hill Road, 1400 West, Flagstaff, AZ 86001.

${ }^{4}$ Sternberg Astronomical Institute, University of Moscow, Universitetskij prosp. 13, Moscow V-234, Russia.

${ }^{5}$ Dominion Astrophysical Observatory, 5071 West Saanich Road, Victoria, B.C., Canada, V8X 4M6.

${ }^{6}$ McDonald Observatory and Department of Astronomy, University of Texas, RLM 15.308, Austin, TX 78712.

${ }^{7}$ Department of Physics and Astronomy, University of Calgary, 2500 University Drive NW, Calgary, AB, Canada, T2N 1 N4.

${ }^{8}$ Crimean Astrophysical Observatory, P/O Nauchny, 334413 Crimea, Ukraine.

${ }^{9}$ Center for Astrophysics and Space Sciences, University of California at San Diego, C-011, La Jolla, CA 92093.

${ }^{10}$ Universitäts-Sternwarte Göttingen, Geismarlandstrasse 11, D-37083 Göttingen, Germany.

${ }_{11}$ Department of Astronomy, University of California, Berkeley, CA 94720.

12 Multiple Mirror Telescope Observatory, University of Arizona, Tucson, AZ 85721.

${ }^{13}$ Deceased.
}

1991, 1992; Dietrich et al. 1993; hereafter Papers II-IV, respectively). By combining the UV and optical results, it has also been possible to study the variability of the "small blue bump," a blend of ultraviolet Fe II and Balmer continuum emission (Maoz et al. 1993). More recently, the southern hemisphere Seyfert 1 galaxy NGC 3783 has been the subject of a similar monitoring effort (Reichert et al. 1994; Stirpe et al. 1994; hereafter Papers V and VI, respectively).

Since the completion of the original monitoring campaign in 1988-89, high temporal resolution, coordinated ground-based monitoring of NGC 5548 has been sustained. The results of the second year of the program are described in Paper III. The primary reasons for continuation of the program are (1) to examine the evolution of the emission-line profiles which

\footnotetext{
${ }^{14}$ Space Telescope Science Institute, 3700 San Martin Drive, Baltimore, MD 21218.

${ }^{15}$ Mailing address: Sterrekundig Instituut, University of Utrecht, P.O. Box 80000, NL-3508 TA, Utrecht, The Netherlands.

${ }^{16}$ Observatories of the Carnegie Institution of Washington, 813 Santa Barbara Street, Pasadena, CA 91101.

${ }^{17}$ Department of Astronomy, University of California, Math-Science Building, Los Angeles, CA 90024.

${ }_{18}$ Osservatorio Astronomico di Bologna, Via Zamboni 33, I-40126, Bologna, Italy.

${ }^{19}$ Institute of Astronomy, Madingley Road, Cambridge CB3 0HZ, United Kingdom.

${ }^{20}$ Royal Greenwich Observatory, Madingley Road, Cambridge CB3 0EZ United Kingdom.

${ }_{21}$ Department of Mathematics, Physics, and Engineering, Mount Royal College, Calgary, Canada, T3E 6K6.

${ }^{22}$ Special Astrophysical Observatory, Russian Academy of Sciences, Nizhni Arkhys, Stavropolsky Kraj, 357140, Russia.

${ }^{23}$ Mailing address: Steward Observatory, University of Arizona, Tucson, AZ 85721 .

${ }_{24}$ Departamento de Astronomia, Instituto de Física, Universidade Federal do Rio Grande do Sul, Avenida Bento Gonçalves, 9500, CP15051, CEP 91500, Porto Alegre, RS, Brazil.
} 
appear to change on timescales longer than the BLR lightcrossing time (and thus probably are not attributable to excitation inhomogeneities in the BLR) and may be due to structural changes in the BLR on the dynamical timescales of a few years, and (2) to study the continuum variability at high temporal resolution in order to determine its short timescale behavior over long time intervals.

In this contribution, we present the first results obtained by doubling the temporal baseline of the published data to $4 \mathrm{yr}$. We present optical continuum and $\mathrm{H} \beta$ emission-line fluxes determined from spectra obtained between 1990 December and 1992 October. The observations are described in $\S 2$, and in $\S 3$ we outline the procedure by which we produce a homogeneous set of continuum and emission-line flux measurments. The results of some preliminary time-series analyses are described in $\S 4$, and our results are summarized in $\S 5$.

\section{OBSERVATIONS}

A complete log of spectroscopic observations appears in Table 1. Columns (1) and (2) give the UT date and Julian date, respectively, of each observation. Column (3) gives a code which indicates the observatory and instrument used to obtain the spectrum; these codes are the same as in Papers II, III, and IV of this series. The projected spectrograph entrance aperture, in arcseconds, is given in column (4). The first dimension is the slit width in the dispersion direction, and the second dimension is the slit length in the cross-dispersion direction; in the case of CCDs, the second entry is the "extraction window" used. The slit position angle is given in column (5), measured in the conventional manner, eastward from north; the cross-dispersion direction runs north-south for a position angle $0^{\circ}$. An estimate of the seeing, when it was recorded at the telescope, is given in column (6). The nominal spectral resolution is given in column (7), and column (8) contains the approximate wavelength range covered by the data. Finally, to aid future investigators who will make use of these data, column (9) gives a unique identifier by which the spectrum is known to the IRAF reduction system, and which is contained in the FITS file header. The file naming convention is the same as used in Papers II and III: the first two characters (" $\mathrm{n} 5$ ") identify the galaxy as NGC 5548, and the next four characters (e.g., "8225") contain the four least significant figures in the Julian date, as in column (2). The next character gives the observatory code, as in column (3). When necessary, an additional arbitrary character is added to eliminate any remaining ambiguity.

\section{ANALYSIS OF THE DATA}

As in Papers II and III, we will at this time restrict our attention only to those spectra in Table 1 which cover the $\mathrm{H} \beta$ spectral region, and discuss only the variability of the optical continuum and the $\mathrm{H} \beta$ emission-line fluxes. Further analysis, including discussion of the emission-line profile variability, will be left to future papers.

\subsection{Absolute Calibration of the Spectra}

As is usual in AGN emission-line studies based on optical spectrophotometry, flux calibration has been carried out by assuming that the narrow emission lines are constant in flux throughout the duration of the monitoring program. The light travel time and recombination time for the extended narrowline region are both sufficiently long that this is almost certainly a valid assumption. In principle, all of the spectra can be placed on a common flux scale by multiplying each spectrum by a constant which is chosen to give the correct integrated flux in the narrow emission lines. The [O III $\lambda 5007$ line is especially useful for flux calibration of spectra of the $\mathrm{H} \beta$ region as it is strong and usually not strongly contaminated by other spectral features. The spectra can be placed on an absolute flux scale if the absolute flux of [O III] $\lambda 5007$ is known. In Paper II, we obtained the absolute flux in the [O III] $\lambda 5007$ line by averaging measurements made through large spectrograph entrance apertures on nights which were reported to be photometric by the observers. In Paper III, we repeated this analysis to ensure that our assumption of constant [O III] $\lambda 5007$ flux is valid. We have again performed this check for the new data reported here. The [O III] $\lambda 5007$ flux, transformed to the rest frame of NGC $5548(z=0.0174)$, is given in Table 2 for those spectra from Table 1 which were obtained through large apertures on photometric nights. These data are in excellent agreement with the measurements for the first $2 \mathrm{yr}$ of the program, as given in Papers II and III. We continue to use the absolute flux given in Paper II $\left(5.58 \times 10^{-13} \mathrm{ergs} \mathrm{s}^{-1} \mathrm{~cm}^{-2}\right)$ in order to keep all of the measurements for all $4 \mathrm{yr}$ on the same flux scale.

\subsection{Spectral Measurements}

Continuum (at $5100 \AA$ in the rest frame of NGC 5548) and $\mathrm{H} \beta$ emission-line measurements were made from the spectra listed in Table 1 as described in Papers II and III. Data with a common origin, as designated by the individual codes in column (3) of Table 1 , are assumed to be homogeneous, except in a few cases where significantly different spectrograph entrance apertures were employed. The flux ratios $F_{\lambda}(5100 \AA) / F([\mathrm{O} \mathrm{III}] \lambda 5007)$ and $F(\mathrm{H} \beta) / F([\mathrm{O} \mathrm{III}] \lambda 5007)$ were measured for each of the spectra in Table 1 in which these features appear. These measured flux ratios are given in Table 3 , grouped by individual homgeneous data set. The continuum and $H \beta$ fluxes can be placed on an absolute scale by multiplying each ratio by the adopted [O III] $\lambda 5007$ flux, i.e., $F_{5007}=5.58 \times 10^{-13} \mathrm{ergs} \mathrm{s}^{-1} \mathrm{~cm}^{-2}$.

\subsection{Intercalibration of the Data}

The larger data sets in Table 3 reveal similar patterns of variability in both the continuum and the $\mathrm{H} \beta$ emission line. However, the light curves produced from the individual sets are slightly offset in flux from one another, and this is attributed to aperture effects. Following Papers II, III, and VI, we can determine an empirical correction for each of the data sets to bring them on to a common flux scale. This is done by adopting one of the larger data sets as a standard, and applying corrections to the other sets that bring measurements from the two sets that are closely spaced in time into agreement. We first define a point-source correction factor $\varphi$ by the equation

$$
F(\mathrm{H} \beta)=\varphi F_{5007}\left[\frac{F(\mathrm{H} \beta)}{F([\mathrm{O} \mathrm{III}] \lambda 5007)}\right]_{\mathrm{obs}},
$$

where $F_{5007}$ is the absolute [O III] $\lambda 5007$ flux, and the observed ratio is as given in Table 3. This factor accounts for the fact that different apertures result in different amounts of light loss for the point-spread function (which describes the surface-brightness distribution of both the broad lines and the AGN continuum source) and the partially extended narrowline region. We note, of course, that this correction factor is in fact a function of seeing. We do not attempt to correct for seeing effects, and this is probably our largest single source of uncertainty. If the surface brightness distribution of the 
TABLE 1

LOG OF SPECTROSCOPIC OBSERVATIONS

\begin{tabular}{|c|c|c|c|c|c|c|c|c|}
\hline \multirow[b]{2}{*}{$\begin{array}{l}\text { UT } \\
\text { DATE } \\
\text { (1) }\end{array}$} & \multirow[b]{2}{*}{$\begin{array}{c}\text { JULIAN DATE } \\
(2,440,000+) \\
\text { (2) }\end{array}$} & \multirow[b]{2}{*}{$\begin{array}{c}\text { CODE } \\
\text { (3) }\end{array}$} & \multicolumn{2}{|c|}{ APERTURE } & \multirow[b]{2}{*}{$\begin{array}{l}\text { SEEING } \\
(") \\
(6)\end{array}$} & \multirow[b]{2}{*}{$\begin{array}{l}\text { REsOLUTION } \\
(\AA) \\
(7)\end{array}$} & \multirow[b]{2}{*}{$\begin{array}{c}\text { RANGE } \\
(\AA) \\
(8)\end{array}$} & \multirow[b]{2}{*}{$\begin{array}{c}\text { IRAF } \\
\text { FILE } \\
(9)\end{array}$} \\
\hline & & & $\begin{array}{l}\text { Size } \\
\text { (4) }\end{array}$ & $\begin{array}{l}\text { P.A. } \\
\text { (5) }\end{array}$ & & & & \\
\hline 1990 Nov 29 & 8225 & D & $4.0 \times 9.0$ & 114 & $1.5-2$ & 8 & $3450-9920$ & $\mathrm{n} 58225 \mathrm{~d}$ \\
\hline $1990 \mathrm{Dec} 5 \ldots$ & 8231 & $\mathrm{~A}$ & $5.0 \times 7.6$ & 90 & 3 & 9 & $4550-5680$ & n58231a \\
\hline 1990 Dec $11 \ldots \ldots \ldots \ldots$ & 8236 & A & $5.0 \times 7.6$ & 90 & 3 & 9 & $4530-5670$ & n58236a \\
\hline 1990 Dec 27 . & 8252 & A & $5.0 \times 7.6$ & 90 & 3 & 9 & $4600-5680$ & n58252a \\
\hline 1991 Jan $6 \ldots$ & 8262 & $\mathbf{H}$ & $4.0 \times 10.0$ & 140 & $1-1.5$ & 20 & $3880-7030$ & $\mathrm{n} 58262 \mathrm{~h}$ \\
\hline 1991 Jan $11 \ldots$ & 8267 & $\mathrm{~A}$ & $5.0 \times 7.6$ & 90 & 4 & 9 & $4600-5670$ & n58267a \\
\hline 1991 Jan 19 ........... & 8275 & A & $5.0 \times 7.6$ & 90 & 3 & 9 & $4600-5650$ & n58275a \\
\hline 1991 Jan 24 ............ & 8280 & A & $5.0 \times 7.6$ & 90 & 3 & 9 & $4520-5670$ & $\mathrm{n} 58280 \mathrm{a}$ \\
\hline 1991 Jan $31 \ldots \ldots \ldots \ldots$ & 8287 & A & $5.0 \times 7.6$ & 90 & 2 & 9 & $4530-5670$ & $\mathrm{n} 58287 \mathrm{a}$ \\
\hline $1991 \mathrm{Feb} 7 \ldots \ldots \ldots \ldots \ldots$ & 8294 & A & $5.0 \times 7.6$ & 90 & 3 & 9 & $4520-5660$ & n58294a \\
\hline 1991 Feb 11 . .......... & 8298 & $\mathrm{~F}$ & $3.2 \times 6.4$ & 90 & $\ldots$ & 5 & $4660-7060$ & $\mathrm{n} 58298 \mathrm{f}$ \\
\hline 1991 Feb 14 .......... & 8301 & $\mathbf{N}$ & $8.8 \times 12.0$ & 0 & $\ldots$ & 12 & $4590-7230$ & n58301n \\
\hline $1991 \mathrm{Feb} 14 \ldots \ldots \ldots \ldots$ & 8302 & $\mathrm{~F}$ & $3.2 \times 6.4$ & 90 & $\ldots$ & 5 & $4660-7060$ & $\mathrm{n} 58302 \mathrm{f}$ \\
\hline 1991 Feb 23 .......... & 8310 & A & $5.0 \times 7.6$ & 90 & 1.5 & 9 & $4520-5660$ & $\mathrm{n} 58310 \mathrm{a}$ \\
\hline 1991 Mar 8 ........... & 8323 & A & $5.0 \times 7.6$ & 90 & 3 & 9 & $4530-5670$ & n58323a \\
\hline 1991 Mar 8 ........... & 8323 & $\mathrm{~F}$ & $3.2 \times 6.4$ & 90 & $\ldots$ & 5 & $4660-7060$ & $\mathrm{n} 58323 \mathrm{f}$ \\
\hline 1991 Mar 9 ........... & 8324 & $\mathrm{~F}$ & $3.2 \times 6.4$ & 90 & $\ldots$ & 5 & $4660-7060$ & n58324f \\
\hline 1991 Mar 10. & 8325 & F & $3.2 \times 6.4$ & 90 & $\ldots$ & 5 & $4660-7060$ & $\mathrm{n} 58325 \mathrm{f}$ \\
\hline 1991 Mar 14 .......... & 8329 & $\mathrm{~F}$ & $3.2 \times 6.4$ & 90 & $\ldots$ & 5 & $4660-7060$ & $\mathrm{n} 58329 \mathrm{f}$ \\
\hline 1991 Mar 15 ........... & 8330 & $\mathbf{F}$ & $3.2 \times 6.4$ & 90 &. & 5 & $4660-7060$ & $\mathrm{n} 58330 \mathrm{f}$ \\
\hline 1991 Mar 23 . . . . . . . & 8338 & A & $5.0 \times 7.6$ & 90 & 2.5 & 9 & $4530-5670$ & n58338a \\
\hline 1991 Mar $26 \ldots \ldots \ldots \ldots$ & 8341 & $\mathrm{~L}$ & 2.0 round & & 4 & 3 & $4100-5140$ & n583411 \\
\hline 1991 Mar 29 . ......... & 8344 & $\overline{\mathbf{A}}$ & $5.0 \times 7.6$ & 90 & 4 & 9 & $4500-5640$ & n58344a \\
\hline 1991 Apr 5 . . . . . . . . & 8351 & A & $5.0 \times 7.6$ & 90 & 3 & 9 & $4510-5650$ & n58351a \\
\hline 1991 Apr 6 . . . . . . . . & 8352 & A & $5.0 \times 7.6$ & 90 & 3 & 9 & $4520-5650$ & $\mathrm{n} 58352 \mathrm{a}$ \\
\hline 1991 Apr 7 .......... & 8353 & $\mathbf{F}$ & $3.2 \times 6.4$ & 90 & & 5 & $4610-7060$ & $\mathrm{n} 58353 \mathrm{f}$ \\
\hline 1991 Apr 7 . . . . . . . & 8354 & $\mathbf{H}$ & $4.0 \times 10.0$ & 60 & 2 & 20 & $3910-6980$ & $\mathrm{n} 58354 \mathrm{~h}$ \\
\hline 1991 Apr 8 . ............ & 8354 & $\mathbf{F}$ & $3.2 \times 6.4$ & 90 & $\ldots$ & 5 & $4610-7060$ & n58354f \\
\hline 1991 Apr 9 & 8355 & $\mathbf{F}$ & $3.2 \times 6.4$ & 90 & $\ldots$ & 5 & $4660-7060$ & n58355f \\
\hline 1991 Apr $10 \ldots \ldots \ldots$ & 8356 & $\mathbf{F}$ & $3.2 \times 6.4$ & 90 & $\ldots$ & 5 & $4720-7060$ & n58356f \\
\hline 1991 Apr 14 .......... & 8360 & $\mathbf{F}$ & $3.2 \times 6.4$ & 90 & $\ldots$ & 5 & $4610-7060$ & $\mathrm{n} 58360 \mathrm{f}$ \\
\hline 1991 Apr 15 .......... & 8361 & $\mathbf{F}$ & $3.2 \times 6.4$ & 90 & $\ldots$ & 5 & $4610-7060$ & n58361f \\
\hline 1991 Apr 16 ........... & 8362 & $\mathbf{F}$ & $3.2 \times 6.4$ & 90 & $\ldots$ & 5 & $4660-7060$ & $\mathrm{n} 58362 \mathrm{f}$ \\
\hline 1991 Apr 19 .......... & 8365 & $\mathrm{~L}$ & 2.0 round & & 4 & 3 & $4070-5100$ & n583651 \\
\hline 1991 Apr 19 ........... & 8365 & A & $5.0 \times 7.6$ & 90 & 2 & 9 & $4520-5660$ & $\mathrm{n} 58365 \mathrm{a}$ \\
\hline 1991 Apr 19 .......... & 8366 & $\mathbf{H}$ & $4.0 \times 10.0$ & 61 & 2 & 20 & $3900-7000$ & n58366h \\
\hline 1991 May 2 ........... & 8378 & $\mathrm{~A}$ & $5.0 \times 7.6$ & 90 & 2 & 9 & $4530-5670$ & n58378a \\
\hline 1991 May $5 \ldots \ldots \ldots \ldots$ & 8381 & $\mathbf{H}$ & $4.0 \times 10.0$ & 241 & 1.3 & 20 & $3910-7000$ & n58381h \\
\hline 1991 May 8 ........... & 8384 & $\mathbf{F}$ & $3.2 \times 6.4$ & 90 & & 5 & $4590-7060$ & n58384f \\
\hline 1991 May $10 \ldots \ldots \ldots \ldots$ & 8386 & A & $5.0 \times 7.6$ & 90 & 4 & 9 & $4520-5660$ & n58386a \\
\hline 1991 May $17 \ldots \ldots \ldots \ldots$ & 8393 & A & $5.0 \times 7.6$ & 90 & 2.5 & 9 & $4520-5660$ & n58393a \\
\hline 1991 May 18 .......... & 8394 & $\mathbf{F}$ & $3.2 \times 6.4$ & 90 & $\ldots$ & 5 & $4580-7060$ & n58394f \\
\hline 1991 May $19 \ldots \ldots \ldots \ldots$ & 8395 & $\mathbf{F}$ & $3.2 \times 6.4$ & 90 & & 5 & $4610-7060$ & n58395f \\
\hline 1991 May $20 \ldots \ldots \ldots \ldots$ & 8396 & L & 2.0 round & & 3 & 3 & $4060-5100$ & n583961 \\
\hline 1991 May $24 \ldots \ldots \ldots \ldots$ & 8400 & A & $5.0 \times 7.6$ & 90 & 1.5 & 9 & $4540-5660$ & $\mathrm{n} 58400 \mathrm{a}$ \\
\hline 1991 Jun 7 ............ & 8414 & A & $5.0 \times 7.6$ & 90 & 3 & 9 & $4530-5670$ & n58414a \\
\hline 1991 Jun 8 ............... & 8415 & $\mathbf{F}$ & $3.2 \times 6.4$ & 90 & & 5 & $4510-7060$ & n58415f \\
\hline 1991 Jun $14 \ldots \ldots \ldots \ldots$ & 8421 & A & $5.0 \times 7.6$ & 90 & 2 & 9 & $4470-5610$ & n58421a \\
\hline 1991 Jun 16 ............ & 8423 & V & $1.2 \times 10.0$ & $\ldots$ & $\ldots$ & 16 & $3200-7980$ & $\mathrm{n} 58423 \mathrm{v}$ \\
\hline 1991 Jun 20 ........... & 8427 & V & $1.2 \times 10.0$ & $\ldots$ & $\ldots$ & 9 & $3500-5970$ & $\mathrm{n} 58427 \mathrm{v}$ \\
\hline 1991 Jun 21 ............ & 8428 & $\mathrm{~V}$ & $1.2 \times 5.0$ & $\ldots$ & & 16 & $3650-8600$ & n58428v \\
\hline 1991 Jun 21 .............. & 8428 & $\mathbf{H}$ & $8.0 \times 7.9$ & 60.1 & 1.5 & 8 & $4270-7300$ & n58428ha \\
\hline 1991 Jun 21 .......... & 8428 & $\mathbf{H}$ & $2.0 \times 8.6$ & 60.1 & 1.5 & 4 & $4540-5330$ & $\mathrm{n} 58428 \mathrm{hb}$ \\
\hline 1991 Jun 21 ........... & 8428 & $\mathbf{H}$ & $2.0 \times 7.9$ & 60.1 & 1.5 & 8 & $6080-7220$ & $\mathrm{n} 58428 \mathrm{hc}$ \\
\hline 1991 Jun 24 ............. & 8431 & A & $5.0 \times 7.6$ & 90 & 2 & 9 & $4530-5670$ & n58431a \\
\hline 1991 Jul 18 ............. & 8455 & A & $5.0 \times 7.6$ & 90 & 2 & 9 & $4520-5650$ & $\mathrm{n} 58455 \mathrm{a}$ \\
\hline 1991 Jul 19 ............ & 8456 & A & $5.0 \times 7.6$ & 90 & 3 & 9 & $4520-5660$ & n58456a \\
\hline 1991 Jul $20 \ldots \ldots \ldots \ldots$ & 8457 & $\mathbf{H}$ & $4.0 \times 10.0$ & 58 & 2 & 20 & $3910-6980$ & $\mathrm{n} 58457 \mathrm{~h}$ \\
\hline 1991 Jul 23 ............ & 8460 & $\mathbf{A}$ & $5.0 \times 7.6$ & 90 & 3 & 9 & $4520-5660$ & n58460a \\
\hline 1991 Aug 5 .............. & 8473 & $\mathbf{H}$ & $4.0 \times 10.0$ & 61 & 4 & 20 & $3910-7040$ & n58473h \\
\hline 1991 Aug 5 ............ & 8474 & $\mathbf{M}$ & $2.0 \times 10.0$ & 90 & 1.5 & 9 & $3950-8100$ & $\mathrm{n} 58474 \mathrm{~m}$ \\
\hline 1991 Aug 8 . ............ & 8477 & $\mathbf{M}$ & $2.0 \times 10.0$ & 90 & 2 & 3 & $4070-5560$ & $\mathrm{n} 58477 \mathrm{~m}$ \\
\hline 1991 Aug 10 .......... & 8479 & $\mathbf{M}$ & $2.0 \times 10.0$ & 90 & 2 & 4 & $5510-7640$ & $\mathrm{n} 58479 \mathrm{n}$ \\
\hline 1991 Aug 20 ........... & 8488 & $\mathbf{H}$ & $4.0 \times 10.0$ & 61 & 1.5 & 20 & $3910-7060$ & $\mathrm{n} 58488 \mathrm{~h}$ \\
\hline 1991 Sep 7 . ............ & 8507 & $\mathbf{M}$ & $1.0 \times 10.0$ & 0 & 1.5 & 20 & $4340-5550$ & $\mathrm{n} 58507 \mathrm{ma}$ \\
\hline 1991 Sep 7 . …........... & 8507 & $\mathbf{M}$ & $1.0 \times 10.0$ & 0 & 1.5 & 15 & $5670-7380$ & $\mathrm{n} 58507 \mathrm{mb}$ \\
\hline 1991 Sep 13 . . . . . . . . & 8512 & A & $5.0 \times 7.6$ & 90 & 2 & 9 & $4560-5660$ & $\mathrm{n} 58512 \mathrm{a}$ \\
\hline 1991 Sep 14 _............. & 8513 & A & $5.0 \times 7.6$ & 90 & 2 & 9 & $4550-5650$ & n58513a \\
\hline 1991 Sep $15 \ldots \ldots \ldots \ldots$ & 8514 & A & $5.0 \times 7.6$ & 90 & $1.5-2$ & 9 & $4550-5650$ & $\mathrm{n} 58514 \mathrm{a}$ \\
\hline
\end{tabular}




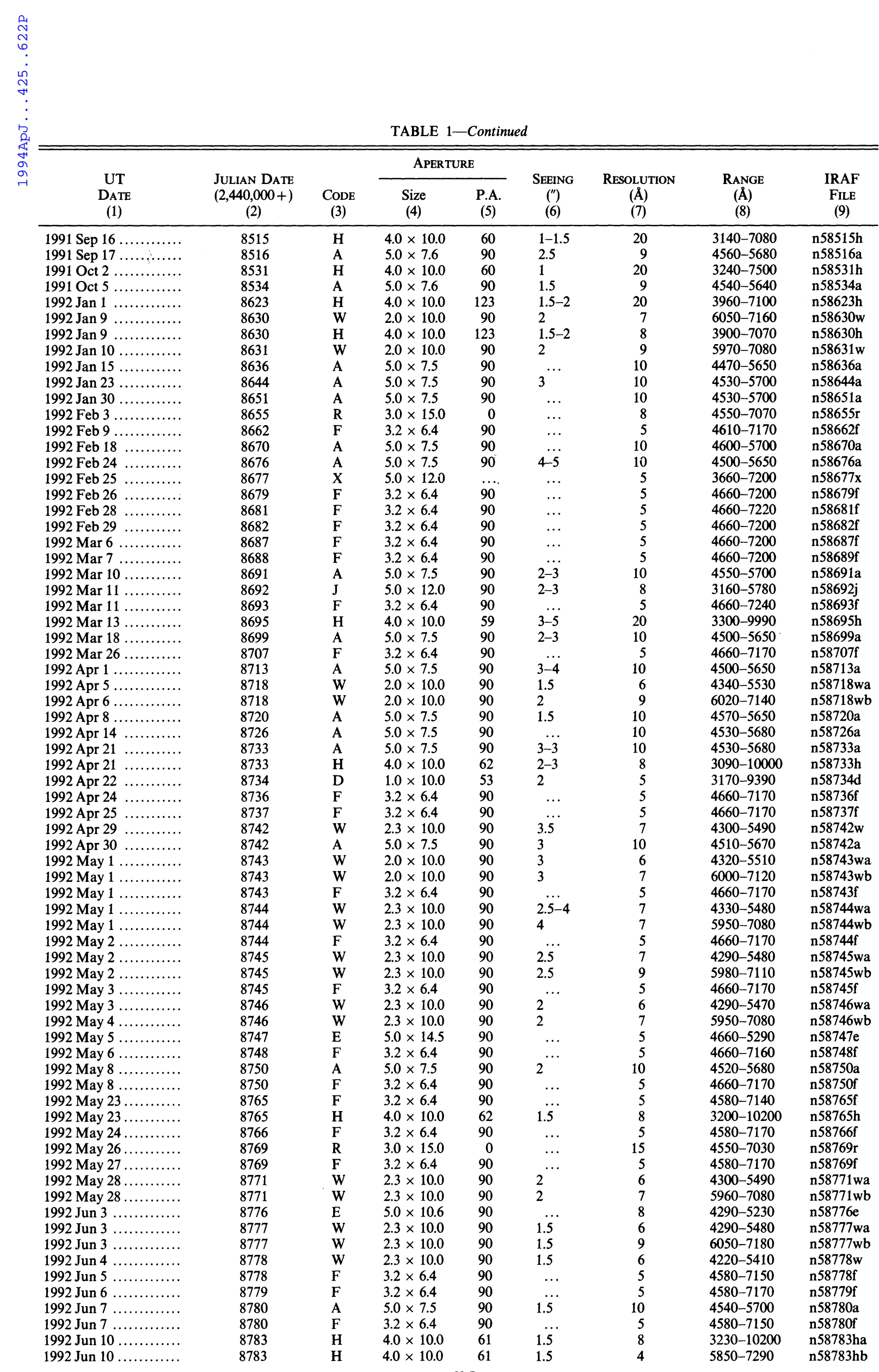


TABLE 1-Continued

\begin{tabular}{|c|c|c|c|c|c|c|c|c|}
\hline \multirow[b]{2}{*}{$\begin{array}{l}\text { UT } \\
\text { DATE } \\
(1)\end{array}$} & \multirow[b]{2}{*}{$\begin{array}{c}\text { JULIAN DATE } \\
(2,440,000+) \\
(2)\end{array}$} & \multirow[b]{2}{*}{$\begin{array}{c}\text { CODE } \\
\text { (3) }\end{array}$} & \multicolumn{2}{|c|}{ APERTURE } & \multirow[b]{2}{*}{$\begin{array}{l}\text { SEEING } \\
(") \\
(6)\end{array}$} & \multirow[b]{2}{*}{$\begin{array}{l}\text { RESOLUTION } \\
(\AA) \\
(7)\end{array}$} & \multirow[b]{2}{*}{$\begin{array}{l}\text { RANGE } \\
(\AA) \\
(8)\end{array}$} & \multirow[b]{2}{*}{$\begin{array}{l}\text { IRAF } \\
\text { FILE } \\
\text { (9) }\end{array}$} \\
\hline & & & $\begin{array}{l}\text { Size } \\
\text { (4) }\end{array}$ & $\begin{array}{c}\text { P.A. } \\
\text { (5) }\end{array}$ & & & & \\
\hline 1992 Jun 16 .. & 8739 & A & $5.0 \times 7.5$ & 90 & 1.5 & 10 & $4550-5710$ & n58789a \\
\hline 1992 Jun 19. & 8793 & $\mathbf{M}$ & 1.3 round & & 3 & 12 & $3820-7340$ & $\mathrm{n} 58793 \mathrm{~m}$ \\
\hline 1992 Jun 23. & 8796 & A & $5.0 \times 7.5$ & 90 & 3 & 10 & $4490-5650$ & n58796a \\
\hline 1992 Jun $26 \ldots$ & 8799 & $\mathrm{~V}$ & $1.2 \times 6.0$ & & 1 & 15 & $4150-9190$ & $\mathrm{n} 58799 \mathrm{v}$ \\
\hline 1992 Jun $28 \ldots \ldots \ldots \ldots$ & 8801 & $\mathrm{~F}$ & $3.2 \times 6.4$ & 90 & & 5 & $4660-7200$ & n58801f \\
\hline 1992 Jun $30 \ldots \ldots \ldots \ldots$ & 8804 & $\mathrm{~W}$ & $2.3 \times 10.0$ & 90 & 2.5 & 7 & $4250-5450$ & n58804w \\
\hline 1992 Jul $1 \ldots \ldots \ldots \ldots$ & 8804 & A & $5.0 \times 7.5$ & 90 & 2 & 10 & $4510-5680$ & $\mathrm{n} 58804 \mathrm{a}$ \\
\hline 1992 Jul $2 \ldots$ & 8805 & $\mathbf{F}$ & $3.2 \times 6.4$ & 90 & $\ldots$ & 5 & $4660-7170$ & $\mathrm{n} 58805 \mathrm{f}$ \\
\hline 1992 Jul $7 \ldots \ldots \ldots \ldots \ldots$ & 8810 & E & $5.0 \times 11.8$ & 90 & & 8 & $4260-5210$ & n58810e \\
\hline 1992 Jul 7 ............. & 8810 & $\mathbf{H}$ & $4.0 \times 10.0$ & 60 & 1.5 & 8 & $3230-10200$ & n58810ha \\
\hline 1992 Jul $7 \ldots$ & 8810 & $\mathbf{H}$ & $4.0 \times 10.0$ & 55 & 1.5 & 4 & $5860-7430$ & $\mathrm{n} 58810 \mathrm{hb}$ \\
\hline 1992 Jul $8 \ldots \ldots \ldots \ldots$ & 8812 & $\mathbf{M}$ & $2.0 \times 10.0$ & 0 & 1.5 & 5 & $3680-5890$ & $\mathrm{n} 58812 \mathrm{ma}$ \\
\hline 1992 Jul $8 \ldots \ldots \ldots \ldots \ldots$ & 8812 & $\mathbf{M}$ & $2.0 \times 10.0$ & 0 & 1.5 & 7 & $5490-9710$ & $\mathrm{n} 58812 \mathrm{mb}$ \\
\hline 1992 Jul $10 \ldots \ldots \ldots \ldots$ & 8813 & E & $5.0 \times 7.2$ & 90 & & 5 & $4740-5210$ & $\mathrm{n} 58813 \mathrm{e}$ \\
\hline 1992 Jul $12 .$. & 8816 & $\mathbf{M}$ & $2.0 \times 10.0$ & 0 & 1 & 5 & $3680-5890$ & $\mathrm{n} 58816 \mathrm{ma}$ \\
\hline 1992 Jul 12 . & 8816 & $\mathbf{M}$ & $2.0 \times 10.0$ & 0 & 1 & 7 & $5510-9260$ & $\mathrm{n} 58816 \mathrm{mb}$ \\
\hline 1992 Jul $14 \ldots \ldots \ldots \ldots$ & 8817 & A & $5.0 \times 7.5$ & 90 & 3-4 & 10 & $4550-5720$ & $\mathrm{n} 58817 \mathrm{a}$ \\
\hline 1992 Jul $15 \ldots \ldots \ldots \ldots$ & 8818 & $\mathrm{E}$ & $5.0 \times 5.8$ & 90 & & 5 & $4690-5160$ & $\mathrm{n} 58818 \mathrm{e}$ \\
\hline 1992 Jul $22 .$. & 8825 & A & $5.0 \times 7.5$ & 90 & $1.5-2$ & 10 & $4540-5710$ & $\mathrm{n} 58825 \mathrm{a}$ \\
\hline 1992 Jul $26 \ldots \ldots \ldots \ldots$ & 8830 & W & $2.0 \times 10.0$ & 90 & 1 & 6 & $4430-5620$ & n58830wa \\
\hline 1992 Jul $26 \ldots \ldots \ldots \ldots$ & 8830 & W & $2.0 \times 10.0$ & 90 & 1 & 7 & $6050-7180$ & $\mathrm{n} 58830 \mathrm{wb}$ \\
\hline 1992 Jul $27 \ldots \ldots \ldots \ldots$ & 8831 & W & $2.0 \times 10.0$ & 90 & 2 & 6 & $4320-5510$ & $\mathrm{n} 58831 \mathrm{w}$ \\
\hline 1992 Jul $28 \ldots \ldots \ldots \ldots$ & 8831 & A & $5.0 \times 7.5$ & 90 & . & 10 & $4580-5750$ & n58831a \\
\hline 1992 Jul $29 \ldots \ldots \ldots \ldots$ & 8833 & W & $2.0 \times 10.0$ & 90 & 3 & 7 & $4350-5540$ & n58833wa \\
\hline 1992 Jul $29 \ldots \ldots \ldots \ldots$ & 8833 & W & $2.0 \times 10.0$ & 90 & 3 & 7 & $6120-7250$ & n58833wb \\
\hline 1992 Jul $30 \ldots \ldots \ldots \ldots$ & 8834 & W & $2.0 \times 10.0$ & 90 & 2.5 & 7 & $4300-5490$ & n58834wa \\
\hline 1992 Jul $30 \ldots \ldots \ldots \ldots$ & 8834 & W & $2.0 \times 10.0$ & 90 & 2.5 & 7 & $6090-7220$ & n58834wb \\
\hline 1992 Jul 31 ............ & 8834 & $\mathrm{E}$ & $5.0 \times 7.2$ & 90 & & 5 & $4710-5180$ & $\mathrm{n} 58834 \mathrm{e}$ \\
\hline 1992 Jul $31 \ldots \ldots \ldots \ldots \ldots$ & 8835 & W & $2.0 \times 10.0$ & 90 & 1 & 7 & $4290-5480$ & $\mathrm{n} 58835 \mathrm{w}$ \\
\hline 1992 Aug 1 . ........... & 8836 & W & $2.3 \times 10.0$ & 90 & 1.5 & 7 & $4240-5440$ & n58836wa \\
\hline 1992 Aug $1 \ldots \ldots \ldots \ldots$ & 8836 & W & $2.3 \times 10.0$ & 90 & 1.5 & 8 & $5970-7100$ & n58836wb \\
\hline 1992 Aug $2 \ldots \ldots \ldots \ldots$ & 8837 & W & $2.3 \times 10.0$ & 90 & 1 & 6 & $4400-5590$ & $\mathrm{n} 58837 \mathrm{w}$ \\
\hline 1992 Aug 3 ........... & 8837 & $\mathbf{H}$ & $4.0 \times 10.0$ & 60 & 1.5 & 8 & $3260-10180$ & $\mathrm{n} 58837 \mathrm{~h}$ \\
\hline 1992 Aug 5 ........... & 8839 & A & $5.0 \times 7.5$ & 90 & $1-2$ & 10 & $4570-5740$ & n58839a \\
\hline 1992 Aug $14 \ldots \ldots \ldots \ldots$ & 8848 & A & $5.0 \times 7.5$ & 90 & $1-2$ & 10 & $4510-5680$ & n58848a \\
\hline 1992 Aug $23 \ldots \ldots \ldots \ldots$ & 8858 & W & $1.5 \times 10.0$ & 90 & 1.5 & 6 & $4300-5500$ & $\mathrm{n} 58858 \mathrm{w}$ \\
\hline 1992 Aug $24 \ldots \ldots \ldots \ldots$ & 8858 & $\mathrm{H}$ & $3.0 \times 7.0$ & 90 & & 12 & $3320-5460$ & $\mathrm{n} 58858 \mathrm{~h}$ \\
\hline 1992 Aug 25 ......... & 8860 & $\mathbf{M}$ & $2.0 \times 10.0$ & 0 & 1 & 10 & $3860-9180$ & $\mathrm{n} 58860 \mathrm{~m}$ \\
\hline 1992 Aug 26 ........... & 8861 & $\mathrm{~W}$ & $1.5 \times 10.0$ & 90 & 1 & 5 & $4340-5520$ & $\mathrm{n} 58861 \mathrm{w}$ \\
\hline 1992 Aug $27 \ldots \ldots \ldots \ldots$ & 8862 & W & $1.5 \times 10.0$ & 90 & 1 & 6 & $4370-5560$ & $\mathrm{n} 58862 \mathrm{w}$ \\
\hline 1992 Aug $28 \ldots \ldots \ldots \ldots$ & 8862 & A & $5.0 \times 7.5$ & 90 & $1-2$ & 10 & $4560-5720$ & $\mathrm{n} 58862 \mathrm{a}$ \\
\hline 1992 Aug $29 \ldots \ldots \ldots$ & 8864 & M & $2.0 \times 10.0$ & 0 & 2 & 10 & $3870-9170$ & $\mathrm{n} 58864 \mathrm{~m}$ \\
\hline 1992 Aug $31 \ldots \ldots \ldots \ldots$ & 8866 & $\mathbf{M}$ & $2.0 \times 10.0$ & 0 & 1 & 10 & $3860-9180$ & $\mathrm{n} 58866 \mathrm{~m}$ \\
\hline 1992 Sep 2 ........... & 8867 & $\mathrm{H}$ & $4.0 \times 10.0$ & 62 & 1.5 & 8 & $3160-10250$ & $\mathrm{n} 58867 \mathrm{~h}$ \\
\hline 1992 Sep 4 & 8869 & A & $5.0 \times 7.5$ & 90 & $1-2$ & 10 & $4530-5690$ & n58869a \\
\hline 1992 Sep $11 \ldots \ldots \ldots \ldots$ & 8876 & A & $5.0 \times 7.5$ & 90 & 2 & 10 & $4580-5730$ & n58876a \\
\hline $1992 \operatorname{Sep} 18 \ldots \ldots \ldots \ldots$ & 8883 & A & $5.0 \times 7.5$ & 90 & & 10 & $4540-5710$ & $\mathrm{n} 58883 \mathrm{a}$ \\
\hline $1992 \operatorname{Sep} 21 \ldots \ldots \ldots \ldots$ & 8886 & $\mathbf{H}$ & $4.0 \times 10.0$ & 61 & 1.5 & 8 & $3160-10250$ & $\mathrm{n} 58886 \mathrm{~h}$ \\
\hline 1992 Sep $24 \ldots \ldots \ldots \ldots$ & 8889 & A & $5.0 \times 7.5$ & 90 & 1.5 & 10 & $4500-5670$ & $\mathrm{n} 58889 \mathrm{a}$ \\
\hline 1992 Oct $3 \ldots \ldots \ldots \ldots$ & 8898 & A & $5.0 \times 7.5$ & 90 & $2-3$ & 10 & $4510-5670$ & n58898a \\
\hline 1992 Oct $3 \ldots \ldots \ldots \ldots$ & 8898 & $\mathbf{H}$ & $4.0 \times 10.0$ & 61 & 2 & 8 & $3200-10250$ & $\mathrm{n} 58898 \mathrm{~h}$ \\
\hline
\end{tabular}

Codes for Data Origin.-A: $1.8 \mathrm{~m}$ Perkins Telescope + Ohio State CCD Spectrograph; D: $5.0 \mathrm{~m}$ Hale Telescope + Double Spectrograph; E: $1.8 \mathrm{~m}$ DAO Telescope + CCD Spectrograph; F: $1.6 \mathrm{~m}$ Mount Hopkins Telescope + Reticon scanner; H: $3.0 \mathrm{~m}$ Shane Telescope + UV Schmidt Spectrograph (through 1992 Jan) + KAST Spectrograph (1992 March and after); J: $2.7 \mathrm{~m}$ McDonald Telescope + Cassegrain Grating Spectrograph; L: $6.0 \mathrm{~m}$ Special Astrophysical Observatory + TV scanner: M: $3.5 \mathrm{~m}$ and $2.2 \mathrm{~m}$ Calar Alto Observatory + CCD spectrographs; N: $1.0 \mathrm{~m}$ Nickel Telescope, Lick Observatory + CCD spectrograph; R: $1.5 \mathrm{~m}$ Loiano Telescope + CCD spectrograph; V: $4.5 \mathrm{~m}$ MMT + CCD spectrograph; W: $2.6 \mathrm{~m}$ Shajn Telescope, Crimean Astrophysical Observatory + CCD spectrograph; X: $1.0 \mathrm{~m}$ CTIO Telescope + 2D-Frutti.

narrow-line region is known, it is indeed possible to model a correction for seeing effects (e.g., Wanders et al. 1992), but there is no indication that such detailed modeling is necessary in the case of NGC 5548, at least for the larger apertures (see, however, Paper IV).

After correcting for aperture effects on the point-spread function to narrow-line ratio, another correction needs to be applied to adjust for the different amounts of starlight admit- ted by different apertures. An extended source correction $G$ is thus defined as

$$
F_{\lambda}(5100 \AA)=\varphi F_{5007}\left[\frac{F_{\lambda}(5100 \AA)}{F([\mathrm{O} \mathrm{III}] \lambda 5007}\right]_{\mathrm{obs}}-G .
$$

The process of intercalibrating the various data sets is then carried out by comparing pairs of nearly simultaneous obser- 
TABLE 2

Absolute Calibration Check

\begin{tabular}{|c|c|}
\hline $\begin{array}{c}F([\mathrm{O} \mathrm{III}] \lambda 5007) \\
\left(10^{-13} \mathrm{ergs} \mathrm{cm}^{-2} \mathrm{~s}^{-1}\right) \\
(1)\end{array}$ & $\begin{array}{l}\text { File Name } \\
\text { (2) }\end{array}$ \\
\hline 5.32 . & $\mathrm{n} 58275 \mathrm{a}$ \\
\hline $5.11 \ldots \ldots \ldots$ & $\mathrm{n} 58287 \mathrm{a}$ \\
\hline $5.30 \ldots \ldots \ldots \ldots \ldots$ & $\mathrm{n} 58393 \mathrm{a}$ \\
\hline $5.61 \ldots \ldots \ldots \ldots \ldots$ & $\mathrm{n} 58414 \mathrm{a}$ \\
\hline $5.61 \ldots \ldots \ldots \ldots \ldots$ & $\mathrm{n} 58473 \mathrm{~h}$ \\
\hline $5.37 \ldots \ldots \ldots \ldots \ldots$ & n58488h \\
\hline $5.41 \ldots \ldots \ldots \ldots \ldots \ldots$ & $\mathrm{n} 58515 \mathrm{~h}$ \\
\hline $5.44 \ldots \ldots \ldots \ldots \ldots \ldots$ & $\mathrm{n} 58530 \mathrm{~h}$ \\
\hline $5.51 \ldots \ldots \ldots$ & $\mathrm{n} 58623 \mathrm{~h}$ \\
\hline $5.23 \ldots$ & $\mathrm{n} 58631 \mathrm{~h}$ \\
\hline $5.32 \ldots \ldots \ldots \ldots \ldots \ldots$ & $\mathrm{n} 58765 \mathrm{~h}$ \\
\hline $5.57 \ldots \ldots \ldots$ & n58783ha \\
\hline $5.60 \ldots \ldots \ldots \ldots \ldots \ldots$ & n58810ha \\
\hline $6.07 \ldots \ldots \ldots \ldots \ldots$ & $\mathrm{n} 58837 \mathrm{~h}$ \\
\hline $5.33 \ldots \ldots \ldots$ & n58898h \\
\hline $5.45 \pm 0.23 \ldots \ldots \ldots$ & $\begin{array}{l}\text { Mean value from } \\
\text { Years 3-4 }\end{array}$ \\
\hline $5.48 \pm 0.24$ & $\begin{array}{l}\text { Mean value from } \\
\text { Year } 2\end{array}$ \\
\hline $5.58 \pm 0.27 \ldots$ & $\begin{array}{l}\text { Mean value from } \\
\text { Year } 1 \text { (adopted } \\
\text { absolute flux) }\end{array}$ \\
\hline
\end{tabular}

vations from different data sets to determine for each data set the values of the constants $\varphi$ and $G$ which are needed to adjust the emission-line and continuum fluxes to a common scale. Furthermore, the formal uncertainties in $\varphi$ and $G$ reflect the uncertainties in the individual data sets, so we can determine the nominal uncertainties for each data set if we assume that the errors added in quadrature. In practice, the interval which we define as "nearly simultaneous" is typically $2-4$ days, which means that any real variability that occurs on timescales this short tends to be somewhat suppressed by the process that allows us to merge the different data sets.

As in Papers II and III, the data are calibrated relative to data set " $A$ " because these data are fairly numerous, overlap reasonably well with most of the other data sets, and were obtained through a reasonably large aperture $(5.0 \times 7$ 7 .6 for the first $3 \mathrm{yr}$, and $5^{\prime \prime} .0 \times 7.5$ for the last year, with the very slight difference attributable to a detector change in autumn $1991)$. Fractional uncertainties of $\sigma_{\text {cont }} / F_{\lambda}(5100 \AA) \approx 0.030$ and $\sigma_{\text {line }} / F(H \beta) \approx 0.040$ for the continuum and $\mathrm{H} \beta$ line, respectively, are adopted for the similar, large-aperture, high-quality data sets " $A$ " and " $H$," based on the differences between closely spaced observations within these sets. These values are very close to those found for the same sets in Papers II and III. The same uncertainties were adopted for the $5 \mathrm{~m}$ Palomar spectrum (set " $D$," large aperture), since these data were obtained in a way nearly identical to the Lick $3 \mathrm{~m}$ data (set " $H$ "). The internal fractional errors of the set " $F$ " data also have been determined from closely spaced observations (i.e., within 2 days), and found to be $\sigma_{\text {cont }} / F_{\lambda}(5100 \AA) \approx 0.050$ and $\sigma_{\text {line }} / F(\mathrm{H} \beta) \approx 0.080$.

For the other data sets, it was possible to estimate the mean uncertainties in the measurements by comparing them to measurements from other sets for which the uncertainties are known and by assuming that the uncertainties for each set add in quadrature.

In Paper IV, it was shown that aperture effects are important for slit sizes smaller than $\sim 2$, and for this reason we have divided the Calar Alto data (set " $M$ ") into two separate sets,

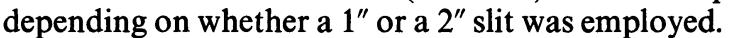

The intercalibration constants we use for each data set are given in Table 4, and these constants are used with equations (1) and (2) to adjust the measurements given in Table 3 to a common flux scale, which corresponds to measurements through the $5.0 \times 7 " .5$ spectrograph entrance aperture used in set "A." The resultant values of the continuum flux $F_{\lambda}(5100 \AA)$ and the line flux $F(\mathrm{H} \beta)$ are given in Table 5, which contains the final light curve produced by computing the variance-weighted average of all the adjusted measurements obtained on a given Julian data.

We can perform a final check of our uncertainty estimates by examining the ratios of all pairs of observations in Table 5 which are separated by 2 days or less. There are 98 independent pairs of measurements within 2 days of one another. The dispersion about the mean (unity), divided by $2^{1 / 2}$, provides an estimate of the typical uncertainty in a single measurement. For the continuum, we find that the mean fractional error in a given measurement is 0.042 . The average fractional uncertainty, from the quoted estimates for these same 98 measurements in Table 5 , is 0.039 , which implies that our error estimates are probably quite good. Examination of the $\mathrm{H} \beta$ emission-line fluxes indicates that the fractional uncertainty is 0.051 , as compared to the mean of the quoted errors for these points, which is 0.056 .

\section{VARIABILITY ANALYSIS}

\subsection{Characteristics of the Data Base}

Figure 1 shows the $5100 \AA$ continuum and $\mathrm{H} \beta$ emission-line light curves from Table 5 . These data span a total of 674 days, with an 89 day gap between the two observing seasons when NGC 5548 is too close to the Sun to observe. In Figure 2, the combined data from Table 5 and Papers II and III are shown. The entire data base covers 1390 days. The 89 day gap between

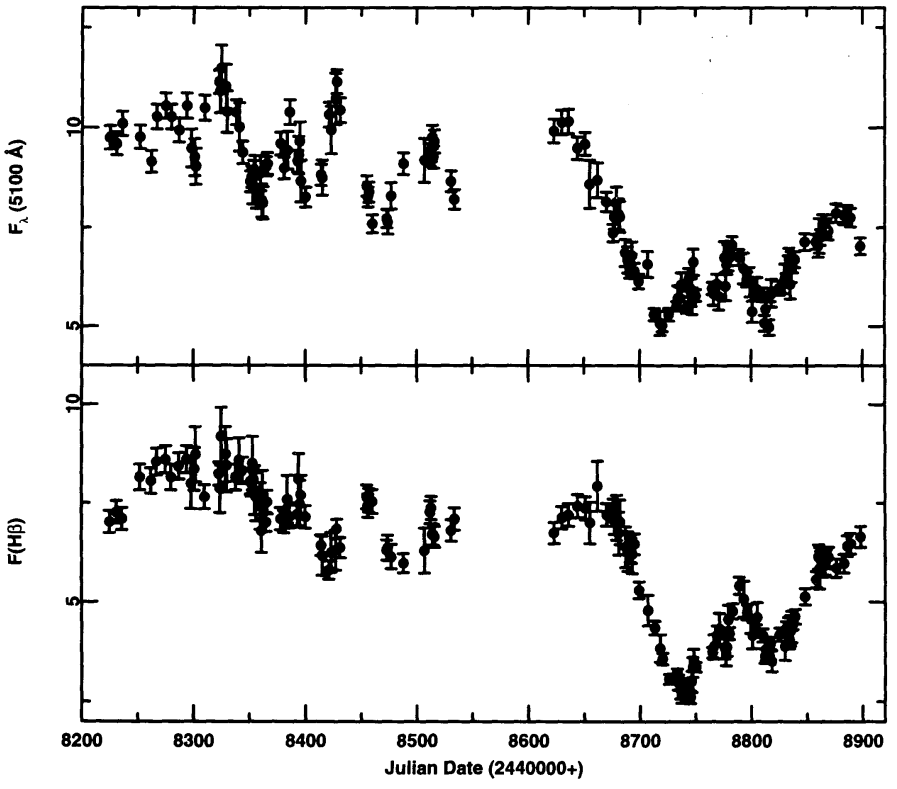

FIG. 1.-The continuum fluxes at $5100 \AA$ (top panel) and $\mathrm{H} \beta$ emission-line fluxes (bottom panel) for NGC 5548, as given in Table 5, from 1990 December to 1992 October. Fluxes are in the rest frame of NGC 5548, and are in units of $10^{-15} \mathrm{ergs} \mathrm{s}^{-1} \mathrm{~cm}^{-2} \AA^{-1}$ for the continuum and $10^{-13} \mathrm{ergs} \mathrm{s}^{-1} \mathrm{~cm}^{-2}$ for the line. 
TABLE 3

MEASUREMENTS OF SPECTRA

\begin{tabular}{|c|c|c|c|c|c|c|c|}
\hline $\begin{array}{c}\text { Julian Date } \\
(2,440,000+) \\
\text { (1) }\end{array}$ & $\frac{100 F_{\lambda}(5100 \AA)}{F\left(\left[\mathrm{O}_{(2)} \lambda 5007\right)\right.}$ & $\frac{F(\mathrm{H} \beta)}{F\left(\left[\begin{array}{l}\mathrm{III}] \\
(3)\end{array} 25007\right)\right.}$ & $\begin{array}{l}\text { IRAF } \\
\text { File } \\
(4)\end{array}$ & $\begin{array}{c}\text { Julian Date } \\
(2,440,000+) \\
\text { (1) }\end{array}$ & $\frac{100 F_{\lambda}(5100 \AA)}{F([\mathrm{O} \mathrm{II}] \lambda 5007)}$ & $\frac{F(\mathrm{H} \beta)}{F([\mathrm{O} \mathrm{II}] \lambda 5007)}$ & $\begin{array}{l}\text { IRAF } \\
\text { File } \\
\text { (4) }\end{array}$ \\
\hline \multicolumn{8}{|c|}{ A-Ohio State CCD } \\
\hline $8231 \ldots \ldots \ldots$ & 1.72 & 1.30 & $\mathrm{n} 58231 \mathrm{a}$ & $8534 .$. & 1.47 & 1.27 & $\mathrm{n} 58534 \mathrm{a}$ \\
\hline $8236 \ldots \ldots \ldots$ & 1.81 & 1.27 & n58236a & $8636 \ldots$ & 1.82 & 1.29 & $\mathrm{n} 58636 \mathrm{a}$ \\
\hline $8252 \ldots$ & 1.75 & 1.46 & n58252a & $8644 \ldots \ldots \ldots$ & 1.70 & 1.33 & $\mathrm{n} 58644 \mathrm{a}$ \\
\hline $8267 \ldots$ & 1.84 & 1.53 & $\mathrm{n} 58267 \mathrm{a}$ & $8651 \ldots \ldots \ldots$ & 1.72 & 1.32 & $\mathrm{n} 58651 \mathrm{a}$ \\
\hline $8275 \ldots \ldots \ldots$ & 1.89 & 1.54 & $\mathrm{n} 58275 \mathrm{a}$ & $8670 \ldots$ & 1.46 & 1.29 & $\mathrm{n} 58670 \mathrm{a}$ \\
\hline $8280 \ldots \ldots \ldots$ & 1.84 & 1.46 & $\mathrm{n} 58280 \mathrm{a}$ & $8676 \ldots$ & 1.32 & 1.31 & $\mathrm{n} 58676 \mathrm{a}$ \\
\hline $8287 \ldots$ & 1.78 & 1.51 & n58287a & $8691 \ldots$ & 1.15 & 1.12 & $\mathrm{n} 58691 \mathrm{a}$ \\
\hline $8294 \ldots \ldots \ldots$ & 1.89 & 1.54 & n58294a & $8699 \ldots$ & 1.10 & 0.95 & $\mathrm{n} 58699 \mathrm{a}$ \\
\hline $8310 \ldots \ldots \ldots$ & 1.88 & 1.37 & $\mathrm{n} 58310 \mathrm{a}$ & $8713 \ldots \ldots \ldots$ & 0.95 & 0.78 & n58713a \\
\hline $8323 \ldots \ldots \ldots$ & 2.02 & 1.46 & n58323a & $8720 \ldots$ & 0.90 & 0.64 & $\mathrm{n} 58720 \mathrm{a}$ \\
\hline $8338 \ldots \ldots \ldots$ & 1.86 & 1.46 & n58338a & $8726 \ldots$ & 0.95 & 0.55 & n58726a \\
\hline $8344 \ldots \ldots \ldots$ & 1.68 & 1.49 & n58344a & $8733 .$. & 0.94 & 0.57 & n58733a \\
\hline $8351 \ldots \ldots \ldots$ & 1.55 & 1.44 & $\mathrm{n} 58351 \mathrm{a}$ & $8742 \ldots$ & 0.96 & 0.54 & $\mathrm{n} 58742 \mathrm{a}$ \\
\hline $8352 \ldots \ldots \ldots$ & 1.57 & 1.43 & $\mathrm{n} 58352 \mathrm{a}$ & $8750 \ldots \ldots \ldots$ & 1.01 & 0.62 & $\mathrm{n} 58750 \mathrm{a}$ \\
\hline $8365 \ldots \ldots \ldots$ & 1.62 & 1.26 & n58365a & $8780 \ldots$ & 1.21 & 0.74 & $\mathrm{n} 58780 \mathrm{a}$ \\
\hline $8378 \ldots \ldots \ldots$ & 1.72 & 1.27 & n58378a & $8789 \ldots$ & 1.21 & 0.97 & $\mathrm{n} 58789 \mathrm{a}$ \\
\hline $8386 \ldots \ldots \ldots$ & 1.86 & 1.28 & $\mathrm{n} 58386 \mathrm{a}$ & $8796 \ldots \ldots \ldots$ & 1.11 & 0.85 & $\mathrm{n} 58796 \mathrm{a}$ \\
\hline $8393 \ldots \ldots \ldots$ & 1.64 & 1.29 & n58393a & $8804 \ldots \ldots \ldots$ & 1.03 & 0.77 & $\mathrm{n} 58804 \mathrm{a}$ \\
\hline $8400 \ldots \ldots \ldots$ & 1.48 & 1.28 & n58400a & $8817 \ldots$ & 1.04 & 0.71 & $\mathrm{n} 58817 \mathrm{a}$ \\
\hline $8414 \ldots \ldots \ldots$ & 1.58 & 1.15 & $\mathrm{n} 58414 \mathrm{a}$ & $8825 \ldots$ & 1.07 & 0.75 & $\mathrm{n} 58825 \mathrm{a}$ \\
\hline $8421 \ldots \ldots \ldots$ & 1.85 & 1.04 & $\mathrm{n} 58421 \mathrm{a}$ & $8831 \ldots \ldots \ldots$ & 1.10 & 0.79 & $\mathrm{n} 58831 \mathrm{a}$ \\
\hline $8431 \ldots \ldots \ldots$ & 1.87 & 1.14 & $\mathrm{n} 58431 \mathrm{a}$ & $8839 \ldots \ldots \ldots$ & 1.20 & 0.83 & $\mathrm{n} 58839 \mathrm{a}$ \\
\hline $8455 \ldots \ldots \ldots$ & 1.53 & 1.37 & $\mathrm{n} 58455 \mathrm{a}$ & $8848 \ldots$ & 1.28 & 0.92 & $\mathrm{n} 58848 \mathrm{a}$ \\
\hline $8456 \ldots \ldots \ldots$ & 1.48 & 1.33 & n58456a & $8862 \ldots \ldots \ldots$ & 1.31 & 1.10 & $\mathrm{n} 58862 \mathrm{a}$ \\
\hline $8460 \ldots \ldots \ldots$ & 1.36 & 1.35 & $\mathrm{n} 58460 \mathrm{a}$ & $8868 \ldots$ & 1.33 & 1.10 & $\mathrm{n} 58869 \mathrm{a}$ \\
\hline $8512 \ldots \ldots \ldots$ & 1.67 & 1.30 & $\mathrm{n} 58512 \mathrm{a}$ & $8876 \ldots \ldots \ldots$ & 1.41 & 1.05 & $\mathrm{n} 58876 \mathrm{a}$ \\
\hline $8513 \ldots \ldots \ldots$ & 1.69 & 1.32 & $\mathrm{n} 58513 \mathrm{a}$ & $8883 \ldots$ & 1.40 & 1.07 & $\mathrm{n} 58883 \mathrm{a}$ \\
\hline $8514 \ldots \ldots \ldots$ & 1.75 & 1.20 & $\mathrm{n} 58514 \mathrm{a}$ & $8889 \ldots \ldots \ldots$ & 1.39 & 1.16 & n58889a \\
\hline $8516 \ldots \ldots \ldots$ & 1.73 & 1.19 & $\mathrm{n} 58516 \mathrm{a}$ & $8898 \ldots$ & 1.26 & 1.19 & n58898a \\
\hline
\end{tabular}

\begin{tabular}{|c|c|c|c|c|c|c|c|}
\hline \multicolumn{8}{|c|}{ D1-Palomar Double Spect. (Large Aperture) } \\
\hline & & $8225 \ldots \ldots \ldots$ & 1.69 & 1.28 & n58225d & & \\
\hline \multicolumn{8}{|c|}{ D2-Palomar Double Spect. (Small Aperture) } \\
\hline & & $8734 \ldots \ldots \ldots$ & 0.66 & 0.70 & n58734d & & \\
\hline \multicolumn{8}{|c|}{ E-DAO CCD } \\
\hline $8747 \ldots \ldots \ldots$ & 0.91 & 0.67 & $\mathrm{n} 58747 \mathrm{e}$ & $8818 \ldots \ldots \ldots$ & 0.95 & 0.70 & $\mathrm{n} 58818 \mathrm{e}$ \\
\hline $8776 \ldots \ldots \ldots$ & 1.13 & 0.77 & n58776e & $8834 \ldots \ldots \ldots$ & 1.11 & 0.80 & n58834e \\
\hline $8813 \ldots \ldots \ldots$ & 0.87 & 0.76 & $\mathrm{n} 58813 \mathrm{e}$ & & & & \\
\hline \multicolumn{8}{|c|}{ F-SAO Reticon } \\
\hline $8298 \ldots \ldots \ldots$ & 1.45 & 1.40 & n58298f & $8682 .$. & 1.15 & 1.23 & $\mathrm{n} 58682 \mathrm{f}$ \\
\hline $8302 \ldots \ldots \ldots$ & 1.37 & 1.53 & $\mathrm{n} 58302 \mathrm{f}$ & $8687 \ldots \ldots \ldots$ & 0.99 & 1.12 & n58687f \\
\hline $8323 \ldots \ldots \ldots$ & 1.68 & 1.51 & n58323f & $8689 .$. & 0.96 & 1.10 & n58689f \\
\hline $8324 \ldots \ldots \ldots$ & 1.70 & 1.38 & n58324f & $8693 .$. & 0.98 & 1.09 & n58693f \\
\hline $8325 \ldots \ldots \ldots$ & 1.80 & 1.61 & $\mathrm{n} 58325 \mathrm{f}$ & $8707 \ldots \ldots \ldots$ & 0.94 & 0.84 & n58707f \\
\hline $8329 \ldots \ldots \ldots$ & 1.72 & 1.53 & n58329f & $8736 \ldots \ldots \ldots$ & 0.79 & 0.49 & n58736f \\
\hline $8330 \ldots \ldots \ldots$ & 1.61 & 1.48 & $\mathrm{n} 58330 \mathrm{f}$ & $8737 \ldots \ldots \ldots$ & 0.85 & 0.47 & n58737f \\
\hline $8353 \ldots \ldots \ldots$ & 1.28 & 1.49 & $\mathrm{n} 58353 \mathrm{f}$ & $8743 \ldots \ldots \ldots$ & 0.87 & 0.42 & $\mathrm{n} 58743 \mathrm{f}$ \\
\hline $8354 \ldots \ldots \ldots$ & 1.27 & 1.40 & n58354f & $8744 \ldots \ldots \ldots$ & 0.85 & 0.50 & $\mathrm{n} 58744 \mathrm{f}$ \\
\hline $8355 \ldots \ldots \ldots$ & 1.34 & 1.37 & $\mathrm{n} 58355 \mathrm{f}$ & $8745 \ldots \ldots \ldots$ & 0.82 & 0.43 & $\mathrm{n} 58745 \mathrm{f}$ \\
\hline $8356 \ldots \ldots \ldots$ & 1.26 & 1.34 & n58356f & $8748 \ldots \ldots \ldots$ & 0.95 & 0.62 & $\mathrm{n} 58748 \mathrm{f}$ \\
\hline $8360 \ldots \ldots \ldots$ & 1.34 & 1.30 & n58360f & $8750 \ldots \ldots \ldots$ & 0.90 & 0.54 & n58750f \\
\hline $8361 \ldots \ldots \ldots$ & 1.22 & 1.19 & n58361f & $8765 \ldots \ldots \ldots$ & 0.83 & 0.60 & n58765f \\
\hline $8362 \ldots \ldots \ldots$ & 1.21 & 1.35 & $\mathrm{n} 58362 \mathrm{f}$ & $8766 \ldots \ldots \ldots$ & 0.81 & 0.68 & $\mathrm{n} 58766 \mathrm{f}$ \\
\hline $8384 \ldots \ldots \ldots$ & 1.44 & 1.33 & n58384f & $8769 .$. & 0.85 & 0.77 & n58769f \\
\hline $8394 \ldots \ldots \ldots$ & 1.42 & 1.42 & n58394f & $8778 \ldots \ldots \ldots$ & 0.96 & 0.70 & n58778f \\
\hline $8395 \ldots \ldots \ldots$ & 1.48 & 1.31 & n58395f & $8779 \ldots \ldots \ldots$ & 0.97 & 0.80 & n58779f \\
\hline $8415 \ldots \ldots \ldots$ & 1.32 & 1.08 & $\mathrm{n} 58415 \mathrm{f}$ & $8780 \ldots \ldots \ldots$ & 0.95 & 0.81 & n58780f \\
\hline $8662 \ldots \ldots \ldots$ & 1.31 & 1.39 & n58662f & $8801, \ldots \ldots \ldots$ & 0.73 & 0.73 & n58801f \\
\hline $8679 \ldots \ldots \ldots$ & 1.21 & 1.25 & $\mathrm{n} 58679 \mathrm{f}$ & $8805^{5} \ldots \ldots \ldots$ & 0.83 & 0.81 & $\mathrm{n} 58805 \mathrm{f}$ \\
\hline $8681 \ldots$ & 1.16 & 1.18 & n58681f & & & & \\
\hline
\end{tabular}


TABLE 3-Continued

\begin{tabular}{|c|c|c|c|c|c|c|c|}
\hline $\begin{array}{c}\text { Julian Date } \\
(2,440,000+) \\
\text { (1) }\end{array}$ & $\frac{100 F_{\lambda}(5100 \AA)}{F\left(\left[\mathrm{O} \mathrm{III}_{(2)} \lambda 5007\right)\right.}$ & $\frac{F(\mathrm{H} \beta)}{F\left(\left[\mathrm{O}_{\text {III }]} \lambda 5007\right)\right.}$ & $\begin{array}{l}\text { IRAF } \\
\text { File } \\
\text { (4) }\end{array}$ & $\begin{array}{c}\text { Julian Date } \\
(2,440,000+) \\
\text { (1) }\end{array}$ & $\frac{100 F_{\lambda}(5100 \AA)}{F\left(\left[\mathrm{O}_{(2)}^{\mathrm{III}]} \lambda 5007\right)\right.}$ & 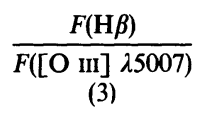 & $\begin{array}{l}\text { IRAF } \\
\text { File } \\
\text { (4) }\end{array}$ \\
\hline \multicolumn{8}{|c|}{$\mathrm{H}$-Lick Shane CCD } \\
\hline $\begin{array}{l}8262 \ldots \ldots \ldots \\
8354 \ldots \ldots \ldots \\
8366 \ldots \ldots \ldots \\
8381 \ldots \ldots \ldots \\
8428 \ldots \ldots \ldots \\
8457 \ldots \ldots \ldots \\
8473 \ldots \ldots \ldots \\
8488 \ldots \ldots \ldots \\
8515 \ldots \ldots \ldots \\
8351 \ldots \ldots \ldots \\
8623 \ldots \ldots \ldots\end{array}$ & $\begin{array}{l}1.58 \\
1.58 \\
1.57 \\
1.55 \\
1.96 \\
1.44 \\
1.32 \\
1.57 \\
1.60 \\
1.49 \\
1.72\end{array}$ & $\begin{array}{l}1.47 \\
1.48 \\
1.37 \\
1.28 \\
1.26 \\
1.38 \\
1.15 \\
1.09 \\
1.22 \\
1.24 \\
1.23\end{array}$ & $\begin{array}{l}\mathrm{n} 58262 \mathrm{~h} \\
\mathrm{n} 58354 \mathrm{~h} \\
\mathrm{n} 58366 \mathrm{~h} \\
\mathrm{n} 58381 \mathrm{~h} \\
\mathrm{n} 58428 \mathrm{ha} \\
\mathrm{n} 58457 \mathrm{~h} \\
\mathrm{n} 58473 \mathrm{~h} \\
\mathrm{n} 58488 \mathrm{~h} \\
\mathrm{n} 58515 \mathrm{~h} \\
\mathrm{n} 58531 \mathrm{~h} \\
\mathrm{n} 58623 \mathrm{~h}\end{array}$ & $\begin{array}{l}8630 \ldots \ldots \ldots \\
8695 \ldots \ldots \ldots \\
8733 \ldots \ldots \ldots \\
8765 \ldots \ldots \ldots \\
8783 \ldots \ldots \ldots \\
8810 \ldots \ldots \ldots \\
8837 \ldots \ldots \ldots \\
8858 \ldots \ldots \ldots \\
8867 \ldots \ldots \ldots \\
8886 \ldots \ldots \ldots \\
8898 \ldots \ldots \ldots \\
\end{array}$ & $\begin{array}{l}1.76 \\
1.08 \\
0.98 \\
1.00 \\
1.20 \\
0.97 \\
1.12 \\
1.22 \\
1.28 \\
1.34 \\
1.22\end{array}$ & $\begin{array}{l}1.30 \\
1.18 \\
0.60 \\
0.70 \\
0.87 \\
0.76 \\
0.83 \\
1.02 \\
1.11 \\
1.17 \\
1.21\end{array}$ & $\begin{array}{l}\text { n58630h } \\
\text { n58695h } \\
\text { n58733h } \\
\text { n58765h } \\
\text { n58783ha } \\
\text { n58810ha } \\
\text { n58837h } \\
\text { n58858h } \\
\text { n58867h } \\
\text { n58886h } \\
\text { n58898h }\end{array}$ \\
\hline \multicolumn{8}{|c|}{ J-McDonald $2.7 \mathrm{~m} \mathrm{CCD}$} \\
\hline & & $8692 \ldots \ldots \ldots$ & 0.86 & 1.11 & $\mathrm{n} 58692 \mathrm{j}$ & & \\
\hline \multicolumn{8}{|c|}{ L-Special Astrophysical Observatory Scanner } \\
\hline $\begin{array}{l}8341 \ldots \ldots \ldots . \\
8365 \ldots \ldots \ldots\end{array}$ & $\begin{array}{l}1.57 \\
1.37\end{array}$ & $\begin{array}{l}1.65 \\
1.33\end{array}$ & $\begin{array}{l}\text { n583411 } \\
\text { n583651 }\end{array}$ & $8396 \ldots \ldots \ldots$ & 1.31 & 1.48 & n583961 \\
\hline \multicolumn{8}{|c|}{ M1-Calar Alto CCD (1" aperture) } \\
\hline $8507 \ldots \ldots \ldots$ & 1.69 & 1.14 & $\mathrm{n} 58507 \mathrm{ma}$ & $8793 \ldots \ldots \ldots$ & 1.20 & 0.92 & $\mathrm{n} 58793 \mathrm{~m}$ \\
\hline \multicolumn{8}{|c|}{ M2-Calar Alto CCD (2" aperture) } \\
\hline $\begin{array}{l}8474 \ldots \ldots \ldots \\
8477 \ldots \ldots \ldots \\
8812 \ldots \ldots \ldots \\
8816 \ldots \ldots \ldots\end{array}$ & $\begin{array}{l}1.25 \\
1.37 \\
0.78 \\
0.76\end{array}$ & $\begin{array}{l}1.17 \\
1.13 \\
0.67 \\
0.67\end{array}$ & $\begin{array}{l}\mathrm{n} 58474 \mathrm{~m} \\
\mathrm{n} 58477 \mathrm{~m} \\
\mathrm{n} 58812 \mathrm{ma} \\
\mathrm{n} 58816 \mathrm{ma}\end{array}$ & $\begin{array}{l}8860 \ldots \ldots \ldots \\
8864 \ldots \ldots \ldots \\
8866 \ldots \ldots \ldots\end{array}$ & $\begin{array}{l}1.14 \\
1.23 \\
1.23\end{array}$ & $\begin{array}{l}1.13 \\
1.09 \\
1.10\end{array}$ & $\begin{array}{l}\mathrm{n} 58860 \mathrm{~m} \\
\mathrm{n} 58864 \mathrm{~m} \\
\mathrm{n} 58866 \mathrm{~m}\end{array}$ \\
\hline \multicolumn{8}{|c|}{$\mathrm{N}$-Lick Nickel CCD } \\
\hline & & $8301 \ldots \ldots \ldots$ & 2.72 & 1.35 & $\mathrm{n} 58301 \mathrm{n}$ & & \\
\hline \multicolumn{8}{|c|}{$\mathrm{R}$-Loiano CCD } \\
\hline $8655 \ldots \ldots \ldots$ & 1.50 & 1.36 & $\mathrm{n} 58655 \mathrm{r}$ & $8769 \ldots \ldots \ldots$ & 1.02 & 0.78 & n58769r \\
\hline \multicolumn{8}{|c|}{$\mathrm{V}-\mathrm{MMT}$ CCD } \\
\hline $\begin{array}{l}8423 \ldots \ldots \ldots \\
8427 \ldots \ldots \ldots\end{array}$ & $\begin{array}{l}1.37 \\
1.51\end{array}$ & $\begin{array}{l}1.13 \\
1.14\end{array}$ & $\begin{array}{l}\text { n58423v } \\
\text { n58427v }\end{array}$ & $\begin{array}{l}8428 \ldots \ldots \ldots \\
8799 \ldots \ldots \ldots\end{array}$ & $\begin{array}{l}1.53 \\
0.68\end{array}$ & $\begin{array}{l}1.19 \\
0.83\end{array}$ & $\begin{array}{l}\mathrm{n} 58428 \mathrm{v} \\
\mathrm{n} 58799 \mathrm{v}\end{array}$ \\
\hline \multicolumn{8}{|c|}{ W-Crimean CCD } \\
\hline $\begin{array}{l}8718 \ldots \ldots \ldots \\
8742 \ldots \ldots \ldots \\
8743 \ldots \ldots \ldots \\
8744 \ldots \ldots \ldots \\
8745 \ldots \ldots \ldots \\
8746 \ldots \ldots \ldots \\
8771 \ldots \ldots \ldots \\
8777 \ldots \ldots \ldots \\
8778 \ldots \ldots \ldots \\
8804 \ldots \ldots \ldots\end{array}$ & $\begin{array}{l}0.74 \\
0.96 \\
0.94 \\
1.03 \\
0.95 \\
0.90 \\
0.88 \\
0.93 \\
1.01 \\
0.97\end{array}$ & $\begin{array}{l}0.78 \\
0.58 \\
0.67 \\
0.61 \\
0.59 \\
0.61 \\
0.88 \\
0.76 \\
0.76 \\
0.85\end{array}$ & $\begin{array}{l}\text { n58718wa } \\
\text { n58742w } \\
\text { n58743wa } \\
\text { n58744wa } \\
\text { n58745wa } \\
\text { n58746wa } \\
\text { n58771wa } \\
\text { n58777wa } \\
\text { n58778w } \\
\text { n58804w }\end{array}$ & $\begin{array}{l}8830 \ldots \ldots \ldots \\
8831 \ldots \ldots \ldots \\
8833 \ldots \ldots \ldots \\
8834 \ldots \ldots \ldots \\
8835 \ldots \ldots \ldots \\
8836 \ldots \ldots \ldots \\
8837 \ldots \ldots \ldots \\
8858 \ldots \ldots \ldots \\
8861 \ldots \ldots \ldots \\
8862 \ldots \ldots \ldots\end{array}$ & $\begin{array}{l}0.97 \\
1.00 \\
1.01 \\
1.08 \\
0.94 \\
1.03 \\
0.97 \\
1.14 \\
1.19 \\
1.14\end{array}$ & $\begin{array}{l}0.79 \\
0.83 \\
0.86 \\
0.84 \\
0.87 \\
0.89 \\
0.84 \\
1.11 \\
1.19 \\
1.17\end{array}$ & $\begin{array}{l}\text { n58830wa } \\
\text { n58831w } \\
\text { n58833wa } \\
\text { n58834wa } \\
\text { n58835w } \\
\text { n58836wa } \\
\text { n58837w } \\
\text { n58858w } \\
\text { n58861w } \\
\text { n58862w }\end{array}$ \\
\hline \multicolumn{8}{|c|}{$\mathrm{X}-$ CTIO 2D-Frutti } \\
\hline & & $8677 \ldots \ldots \ldots$ & 1.40 & 1.25 & $\mathrm{n} 58677 \mathrm{x}$ & & \\
\hline
\end{tabular}

the third and fourth year is by far the largest gap in the coverage; the gaps between the first and second year and between the second and third year are only 52 days and 46 days, respectively. The general characteristics of the data base are summarized in Table $6{ }^{25}$ The fractional variation $F_{\mathrm{var}}$ is the ratio

${ }^{25}$ The sampling characteristics given in Table 6 supersede the incorrect values given in Paper III. of the rms fluctuation to the mean flux and is corrected for the effect of measurement errors (see Paper I). The parameter $R_{\max }$ is the ratio of maximum to minimum flux. Neither of these parameters has been adjusted for the effects of nonvarying components, such as the stellar continuum or the $\mathrm{H} \beta$ narrow line.

Inspection of Figure 2 shows that the character of the continuum variations was considerably different during the third 
TABLE 4

FLUX SCALE FaCtors

\begin{tabular}{|c|c|c|}
\hline $\begin{array}{l}\text { Data } \\
\text { Set } \\
\text { (1) }\end{array}$ & $\begin{array}{c}\text { Point-Source } \\
\text { Scale Factor } \\
\varphi \\
(2)\end{array}$ & $\begin{array}{c}\text { Extended Source } \\
\text { Correction } G \\
\left(10^{-15} \mathrm{ergs} \mathrm{s}^{-1}\right. \\
\left.\mathrm{cm}^{-2} \AA^{-1}\right) \\
\text { (3) }\end{array}$ \\
\hline A & 1.000 & 0.000 \\
\hline D1 & 0.982 & -0.491 \\
\hline & $0.764 \pm 0.075$ & $-2.906 \pm 0.347$ \\
\hline & $0.900 \pm 0.102$ & $-1.075 \pm 0.088$ \\
\hline & $1.022 \pm 0.085$ & $-1.214 \pm 0.497$ \\
\hline $\mathrm{H}$. & $0.982 \pm 0.042$ & $-0.491 \pm 0.337$ \\
\hline $\mathbf{J} \ldots$ & $1.017 \pm 0.018$ & $-1.697 \pm 0.197$ \\
\hline & $0.932 \pm 0.060$ & $-1.846 \pm 0.646$ \\
\hline M1 ........... & $0.989 \pm 0.092$ & $0.150 \pm 0.395$ \\
\hline M2. & $0.974 \pm 0.060$ & $-0.843 \pm 0.381$ \\
\hline & $1.109 \pm 0.070$ & $7.578 \pm 0.322$ \\
\hline R ... & $0.922 \pm 0.085$ & $-0.869 \pm 0.505$ \\
\hline V. & $0.990 \pm 0.067$ & $-2.369 \pm 0.479$ \\
\hline $\mathrm{W}$. & $0.882 \pm 0.118$ & $-1.439 \pm 0.427$ \\
\hline & $1.012 \pm 0.043$ & $0.132 \pm 0.378$ \\
\hline
\end{tabular}

year (1991) of this program than it has been at other times. Compared to the other years in which NGC 5548 has been closely monitored, the continuum variations was more rapid and of lower amplitude. This behavior is reminiscent of that observed in NGC 3783 (Papers V and VI) and shows very clearly that the nature of the continuum variations can change over time.

The fourth year (1992) of this program is also notable in that NGC 5548 reached its faintest recorded state. This has also been reported by Iijima, Rafanelli, \& Bianchini (1992). A sample spectrum obtained during this very low state is shown in Figure 3, along with a high-state spectrum from Paper I. The

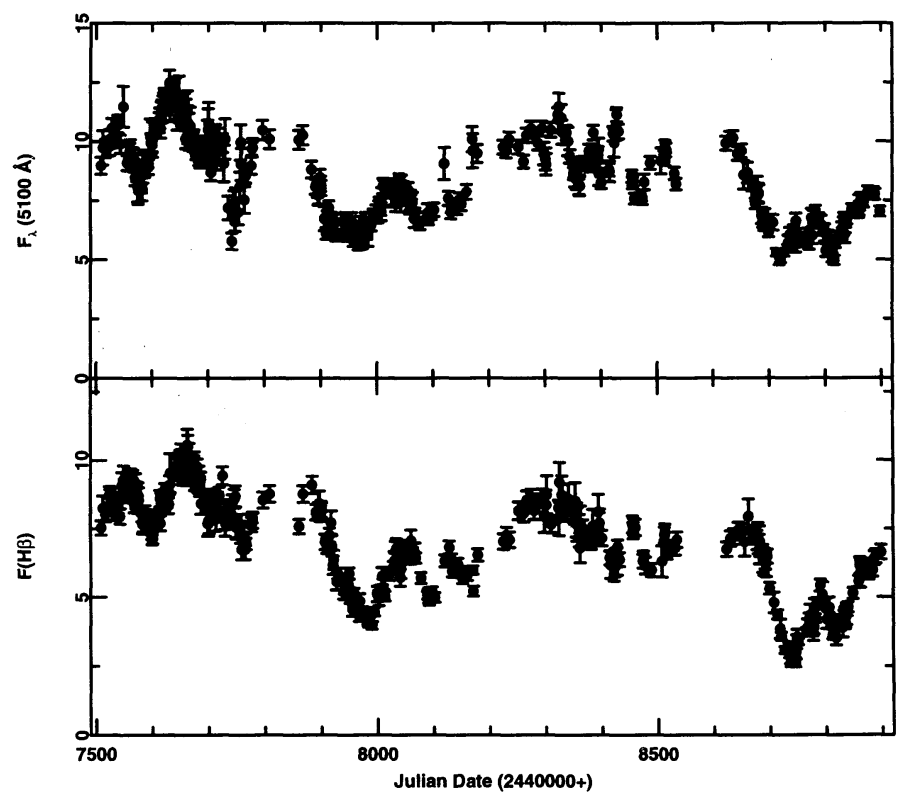

FIG. 2.-Optical continuum $(5100 \AA)$ and $\mathrm{H} \beta$ emission-line light curves are shown for all $4 \mathrm{yr}$ of the NGC 5548 monitoring campaign. The data for the first year (1988 December-1989 October) and the second year (1989 December1990 October) are from Tables A2 and 8, respectively, from Paper III, and the rest of the data are as in Table 5 and Fig. 1. Fluxes are in the rest frame of NGC 5548, and are in units of $10^{-15}$ ergs s$~^{-1} \mathrm{~cm}^{-2} \AA^{-1}$ for the continuum and $10^{-13} \mathrm{ergs} \mathrm{s}^{-1} \mathrm{~cm}^{-2}$ for the line.

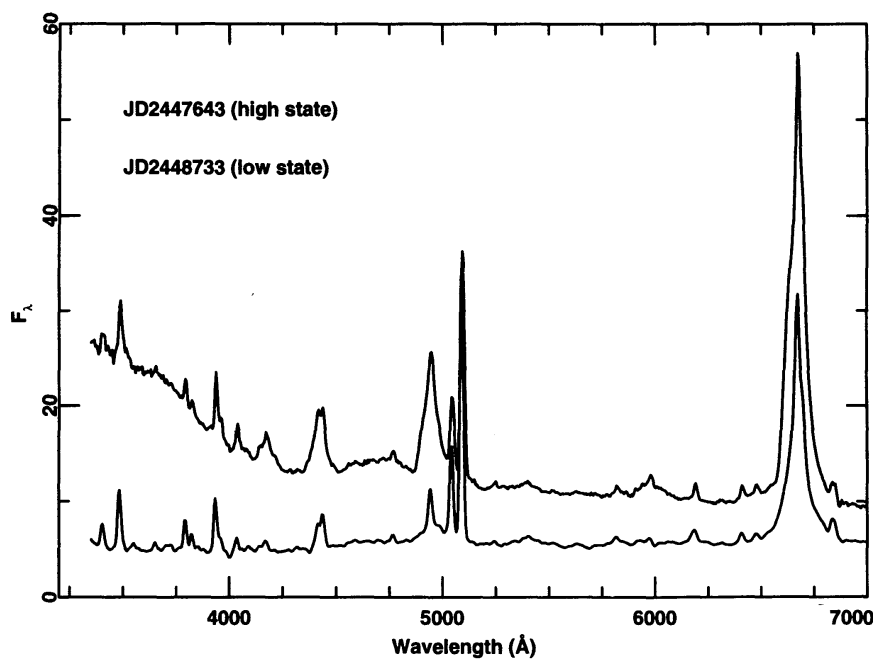

Fig. 3.-Two optical spectra, both obtained with the Lick $3 \mathrm{~m}$ Shane telescope, showing high and low states of NGC 5548. The lower spectrum was obtained on JD 2,448,733 (1992 May 1). The upper spectrum, from Paper I, was obtained on JD 2,447,643 (1989 April 27). Fluxes are in units of $10^{-15}$ ergs $\mathrm{s}^{-1} \mathrm{~cm}^{-2} \AA^{-1}$ and are shown in the observed frame. The long wavelength end of the "small blue bump," which is prominent below $4100 \AA$ in the high-state spectrum, is virtually absent in the low-state spectrum.

data obtained during this low state will be of particular use in determining both the narrow-line contributions to the broad emission features, and the starlight contribution to the optical spectra. This will be done in a future paper.

\subsection{Time-Series Analysis}

The lag between variations of the optical continuum and the $\mathrm{H} \beta$ emission-line response can be determined by cross correlation of the two light curves. Unfortunately, cross-correlation analysis of unevenly sampled series is not completely straightforward. Here we adjust for the irregular sampling by using two methods that are commonly employed in AGN variability studies, the interpolation method of Gaskell \& Sparke (1986) and Gaskell \& Peterson (1987), and the discrete correlation function (DCF) method of Edelson \& Krolik (1988), in both cases with some minor modifications.

In the interpolation method, regular sampling is achieved by interpolating the light curves one at a time in a piecewise linear fashion between the real observations. The value of the crosscorrelation function (CCF) is computed for any arbitrary lag $\tau$ by pairing each of the real data points in the emission-line light curve $L\left(t_{i}\right)$ with interpolated points in the continuum light curve $C\left(t_{i}-\tau\right)$ for all points which are within the real limits of each light curve. The CCF is then computed a second time, this time interpolating in the other series, pairing this time the real points $C\left(t_{j}\right)$ with the interpolated points $L\left(t_{j}+\tau\right)$. The results of these two computations are then averaged to form the final CCF. For the cross correlations performed here, we normalize the amplitude of the CCF at each lag by using the mean and variance computed only from those points which contribute to the CCF at that particular lag. This is in contrast to the usual practice of normalizing with the mean and variance for the whole time series (in other words, we are not assuming that the light curves are statistically stationary).

In the DCF method, problem of uneven sampling is dealt with by computing the correlation coefficient at lag $\tau$ using only real pairs of data separated by intervals in the range 
TABLE 5

Optical ContinuUm and H $\beta$ Light Curves

\begin{tabular}{|c|c|c|c|c|c|}
\hline $\begin{array}{c}\text { Julian Date } \\
(2,440,000+) \\
(1)\end{array}$ & $\begin{array}{c}F_{\lambda}(5100 \AA) \\
\left(10^{-15} \mathrm{ergs} \mathrm{s}^{-1}\right. \\
\left.\mathrm{cm}^{-2} \AA^{-1}\right) \\
(2)\end{array}$ & $\begin{array}{c}F(\mathrm{H} \beta) \\
\left(10^{-13} \text { ergs }\right. \\
\left.\mathrm{s}^{-1} \mathrm{~cm}^{-2}\right) \\
(3)\end{array}$ & $\begin{array}{c}\text { Julian Date } \\
(2,440,000+) \\
(1)\end{array}$ & $\begin{array}{c}F_{\lambda}(5100 \AA) \\
\left(10^{-15} \mathrm{ergs} \mathrm{s}^{-1}\right. \\
\left.\mathrm{cm}^{-2} \AA^{-1}\right) \\
(2)\end{array}$ & $\begin{array}{c}F(\mathrm{H} \beta) \\
\left(10^{-13} \text { ergs }\right. \\
\left.\mathrm{s}^{-1} \mathrm{~cm}^{-2}\right) \\
(3)\end{array}$ \\
\hline 8225 . & $9.75 \pm 0.29$ & $7.01 \pm 0.28$ & 8651 & $9.60 \pm 0.29$ & $7.37 \pm 0.29$ \\
\hline 8231. & $9.60 \pm 0.29$ & $7.25 \pm 0.29$ & 8655 & $8.59 \pm 0.60$ & $7.00 \pm 0.52$ \\
\hline 8236. & $10.10 \pm 0.30$ & $7.09 \pm 0.28$ & 8662 . & $8.69 \pm 0.43$ & $7.93 \pm 0.63$ \\
\hline 8252. & $9.77 \pm 0.29$ & $8.15 \pm 0.33$ & 8670 . & $8.15 \pm 0.24$ & $7.20 \pm 0.29$ \\
\hline 8262 . & $9.15 \pm 0.27$ & $8.06 \pm 0.32$ & 8676 . & $7.37 \pm 0.22$ & $7.31 \pm 0.29$ \\
\hline 8267. & $10.27 \pm 0.31$ & $8.54 \pm 0.34$ & 8677. & $7.77 \pm 0.31$ & $7.06 \pm 0.35$ \\
\hline 8275 . & $10.55 \pm 0.32$ & $8.59 \pm 0.34$ & 8679 . & $8.11 \pm 0.41$ & $7.13 \pm 0.57$ \\
\hline 8280 . & $10.27 \pm 0.31$ & $8.15 \pm 0.33$ & 8681 . & $7.83 \pm 0.39$ & $6.73 \pm 0.54$ \\
\hline 8287. & $9.93 \pm 0.30$ & $8.43 \pm 0.34$ & 8682 . & $7.77 \pm 0.39$ & $7.01 \pm 0.56$ \\
\hline 8294. & $10.55 \pm 0.32$ & $8.59 \pm 0.34$ & 8687. & $6.86 \pm 0.34$ & $6.39 \pm 0.51$ \\
\hline 8298. & $9.48 \pm 0.47$ & $7.98 \pm 0.64$ & 8689. & $6.69 \pm 0.33$ & $6.27 \pm 0.50$ \\
\hline 8301. & $9.25 \pm 0.46$ & $8.35 \pm 0.54$ & 8691 . & $6.42 \pm 0.19$ & $6.25 \pm 0.25$ \\
\hline 8302 . & $9.03 \pm 0.45$ & $8.73 \pm 0.70$ & 8692 . & $6.58 \pm 0.26$ & $6.30 \pm 0.31$ \\
\hline 8310 . & $10.49 \pm 0.31$ & $7.64 \pm 0.31$ & 8693. & $6.80 \pm 0.34$ & $6.22 \pm 0.50$ \\
\hline 8323. & $11.14 \pm 0.29$ & $8.23 \pm 0.29$ & 8695 . & $6.41 \pm 0.19$ & $6.47 \pm 0.26$ \\
\hline $8324 \ldots \ldots \ldots$ & $10.91 \pm 0.55$ & $7.87 \pm 0.63$ & 8699. & $6.14 \pm 0.18$ & $5.30 \pm 0.21$ \\
\hline $8325 \ldots \ldots \ldots$ & $11.48 \pm 0.57$ & $9.18 \pm 0.74$ & 8707. & $6.57 \pm 0.33$ & $4.79 \pm 0.38$ \\
\hline 8329. & $11.02 \pm 0.55$ & $8.73 \pm 0.70$ & 8713. & $5.30 \pm 0.16$ & $4.35 \pm 0.17$ \\
\hline 8330 . & $10.40 \pm 0.52$ & $8.44 \pm 0.68$ & 8718. & $5.08 \pm 0.31$ & $3.84 \pm 0.34$ \\
\hline 8338. & $10.38 \pm 0.31$ & $8.15 \pm 0.33$ & 8720 . & $5.02 \pm 0.15$ & $3.57 \pm 0.14$ \\
\hline 8341. & $10.01 \pm 0.60$ & $8.58 \pm 0.56$ & 8726 . & $5.30 \pm 0.16$ & $3.07 \pm 0.12$ \\
\hline 8344. & $9.37 \pm 0.28$ & $8.31 \pm 0.33$ & 8733. & $5.52 \pm 0.12$ & $3.23 \pm 0.09$ \\
\hline 8351. & $8.65 \pm 0.26$ & $8.03 \pm 0.32$ & 8734. & $5.72 \pm 0.31$ & $2.98 \pm 0.19$ \\
\hline $8352 \ldots \ldots \ldots$ & $8.76 \pm 0.26$ & $7.98 \pm 0.32$ & 8736 . & $5.72 \pm 0.29$ & $2.79 \pm 0.22$ \\
\hline $8353 \ldots \ldots \ldots$ & $8.51 \pm 0.43$ & $8.50 \pm 0.68$ & 8737. & $6.06 \pm 0.30$ & $2.68 \pm 0.21$ \\
\hline 8354. & $8.94 \pm 0.23$ & $8.08 \pm 0.29$ & 8742 . & $5.49 \pm 0.15$ & $2.98 \pm 0.11$ \\
\hline 8355 . & $8.85 \pm 0.44$ & $7.81 \pm 0.63$ & 8743. & $6.13 \pm 0.24$ & $2.66 \pm 0.16$ \\
\hline 8356. & $8.40 \pm 0.42$ & $7.64 \pm 0.61$ & 8744. & $6.23 \pm 0.24$ & $2.91 \pm 0.17$ \\
\hline $8360 \ldots \ldots \ldots$ & $8.85 \pm 0.44$ & $7.41 \pm 0.59$ & 8745 . & $5.98 \pm 0.23$ & $2.62 \pm 0.16$ \\
\hline 8361 . & $8.17 \pm 0.41$ & $6.79 \pm 0.54$ & 8746. & $5.87 \pm 0.35$ & $3.00 \pm 0.27$ \\
\hline 8362 . & $8.11 \pm 0.41$ & $7.70 \pm 0.62$ & 8747. & $5.64 \pm 0.34$ & $3.37 \pm 0.25$ \\
\hline $8365 \ldots \ldots \ldots$ & $9.03 \pm 0.24$ & $7.00 \pm 0.24$ & 8748. & $6.63 \pm 0.33$ & $3.54 \pm 0.28$ \\
\hline $8366 \ldots \ldots \ldots$ & $9.09 \pm 0.27$ & $7.51 \pm 0.30$ & $8750 .$. & $5.79 \pm 0.15$ & $3.37 \pm 0.12$ \\
\hline 8378 . & $9.60 \pm 0.29$ & $7.09 \pm 0.28$ & $8765 \ldots$ & $5.96 \pm 0.15$ & $3.74 \pm 0.13$ \\
\hline 8381 . & $8.98 \pm 0.27$ & $7.01 \pm 0.28$ & 8766 . & $5.83 \pm 0.29$ & $3.88 \pm 0.31$ \\
\hline $8384 \ldots \ldots \ldots$ & $9.43 \pm 0.47$ & $7.58 \pm 0.61$ & 8769. & $6.08 \pm 0.25$ & $4.17 \pm 0.23$ \\
\hline $8386 \ldots \ldots \ldots$ & $10.38 \pm 0.31$ & $7.14 \pm 0.29$ & 8771. & $5.77 \pm 0.35$ & $4.33 \pm 0.39$ \\
\hline 8393. & $9.15 \pm 0.28$ & $7.20 \pm 0.29$ & 8776 . & $6.75 \pm 0.41$ & $3.87 \pm 0.29$ \\
\hline 8394. & $9.31 \pm 0.47$ & $8.10 \pm 0.65$ & 8777. & $6.02 \pm 0.36$ & $3.74 \pm 0.34$ \\
\hline 8395. & $9.65 \pm 0.48$ & $7.47 \pm 0.60$ & 8778 . & $6.57 \pm 0.25$ & $3.87 \pm 0.23$ \\
\hline $8396 \ldots \ldots \ldots$ & $8.66 \pm 0.52$ & $7.70 \pm 0.50$ & $8779 \ldots$ & $6.75 \pm 0.34$ & $4.56 \pm 0.37$ \\
\hline 8400. & $8.26 \pm 0.25$ & $7.14 \pm 0.29$ & $8780 .$. & $6.72 \pm 0.17$ & $4.21 \pm 0.15$ \\
\hline 8414 . & $8.82 \pm 0.26$ & $6.42 \pm 0.26$ & 8783. & $7.07 \pm 0.21$ & $4.77 \pm 0.19$ \\
\hline $8415 \ldots \ldots \ldots$ & $8.74 \pm 0.44$ & $6.16 \pm 0.49$ & 8789. & $6.75 \pm 0.20$ & $5.41 \pm 0.22$ \\
\hline $8421 \ldots \ldots \ldots$ & $10.32 \pm 0.31$ & $5.80 \pm 0.23$ & 8793. & $6.47 \pm 0.39$ & $5.08 \pm 0.46$ \\
\hline 8423. & $9.94 \pm 0.60$ & $6.24 \pm 0.50$ & $8796 .$. & $6.19 \pm 0.19$ & $4.74 \pm 0.19$ \\
\hline 8427. & $10.71 \pm 0.64$ & $6.30 \pm 0.50$ & $8799 .$. & $6.13 \pm 0.37$ & $4.59 \pm 0.37$ \\
\hline 8428 . & $11.14 \pm 0.30$ & $6.83 \pm 0.24$ & $8801 \ldots$ & $5.38 \pm 0.27$ & $4.16 \pm 0.33$ \\
\hline 8431. & $10.44 \pm 0.31$ & $6.36 \pm 0.25$ & $8804 .$. & $5.83 \pm 0.16$ & $4.28 \pm 0.16$ \\
\hline 8455. & $8.54 \pm 0.26$ & $7.64 \pm 0.31$ & $8805 \ldots$ & $5.95 \pm 0.30$ & $4.62 \pm 0.37$ \\
\hline 8456 . & $8.26 \pm 0.25$ & $7.42 \pm 0.30$ & $8810 .$. & $5.81 \pm 0.17$ & $4.16 \pm 0.17$ \\
\hline $8457 .$. & $8.38 \pm 0.25$ & $7.56 \pm 0.30$ & $8812 \ldots$ & $5.08 \pm 0.20$ & $3.64 \pm 0.18$ \\
\hline 8460. & $7.59 \pm 0.23$ & $7.53 \pm 0.30$ & 8813. & $5.44 \pm 0.33$ & $3.82 \pm 0.29$ \\
\hline 8473 . & $7.72 \pm 0.23$ & $6.30 \pm 0.25$ & $8816 \ldots$ & $4.97 \pm 0.20$ & $3.64 \pm 0.18$ \\
\hline 8474. & $7.64 \pm 0.31$ & $6.36 \pm 0.32$ & $8817 \ldots$ & $5.80 \pm 0.17$ & $3.96 \pm 0.16$ \\
\hline $8477 \ldots$ & $8.29 \pm 0.33$ & $6.14 \pm 0.31$ & $8818 \ldots$ & $5.85 \pm 0.35$ & $3.52 \pm 0.26$ \\
\hline $8488 .$. & $9.09 \pm 0.27$ & $5.97 \pm 0.24$ & $8825 \ldots$ & $5.97 \pm 0.18$ & $4.18 \pm 0.17$ \\
\hline $8507 .$. & $9.18 \pm 0.55$ & $6.29 \pm 0.57$ & $8830 \ldots$ & $6.21 \pm 0.37$ & $3.89 \pm 0.35$ \\
\hline $8512 \ldots$ & $9.32 \pm 0.28$ & $7.25 \pm 0.29$ & $8831 \ldots$ & $6.18 \pm 0.17$ & $4.35 \pm 0.16$ \\
\hline $8513 \ldots$ & $9.43 \pm 0.28$ & $7.37 \pm 0.29$ & $8833 \ldots$ & $6.41 \pm 0.38$ & $4.23 \pm 0.38$ \\
\hline 8514. & $9.77 \pm 0.29$ & $6.70 \pm 0.27$ & $8834 \ldots$ & $6.70 \pm 0.28$ & $4.06 \pm 0.23$ \\
\hline $8515 \ldots$ & $9.26 \pm 0.28$ & $6.68 \pm 0.27$ & $8835 \ldots$ & $6.07 \pm 0.36$ & $4.28 \pm 0.38$ \\
\hline $8516 \ldots$ & $9.65 \pm 0.29$ & $6.64 \pm 0.27$ & 8836. & $6.51 \pm 0.39$ & $4.38 \pm 0.39$ \\
\hline $8531 \ldots$ & $8.65 \pm 0.26$ & $6.79 \pm 0.27$ & $8837 .$. & $6.54 \pm 0.18$ & $4.47 \pm 0.16$ \\
\hline $8534 \ldots$ & $8.20 \pm 0.25$ & $7.09 \pm 0.28$ & $8839 \ldots$ & $6.70 \pm 0.20$ & $4.63 \pm 0.19$ \\
\hline $8623 \ldots$ & $9.92 \pm 0.30$ & $6.74 \pm 0.27$ & $8848 \ldots$ & $7.14 \pm 0.21$ & $5.13 \pm 0.20$ \\
\hline 8630. & $10.14 \pm 0.30$ & $7.12 \pm 0.28$ & $8858 \ldots$ & $7.15 \pm 0.19$ & $5.57 \pm 0.20$ \\
\hline 8636. & $10.16 \pm 0.31$ & $7.20 \pm 0.29$ & $8860 .$. & $7.04 \pm 0.28$ & $6.14 \pm 0.31$ \\
\hline $8644 .$. & $9.49 \pm 0.28$ & $7.42 \pm 0.30$ & 8861. & $7.30 \pm 0.44$ & $5.86 \pm 0.53$ \\
\hline
\end{tabular}


TABLE 5-Continued

\begin{tabular}{|c|c|c|c|c|c|}
\hline $\begin{array}{c}\text { Julian Date } \\
(2,440,000+) \\
\text { (1) }\end{array}$ & $\begin{array}{c}F_{\lambda}(5100 \AA) \\
\left(10^{-15} \mathrm{ergs} \mathrm{s}^{-1}\right. \\
\left.\mathrm{cm}^{-2} \AA^{-1}\right) \\
(2)\end{array}$ & $\begin{array}{c}F(\mathrm{H} \beta) \\
\left(10^{-13} \mathrm{ergs}\right. \\
\left.\mathrm{s}^{-1} \mathrm{~cm}^{-2}\right) \\
(3)\end{array}$ & $\begin{array}{c}\text { Julian Date } \\
(2,440,000+) \\
\text { (1) }\end{array}$ & $\begin{array}{c}F_{\lambda}(5100 \AA) \\
\left(10^{-15} \mathrm{ergs} \mathrm{s}^{-1}\right. \\
\left.\mathrm{cm}^{-2} \AA^{-1}\right) \\
(2)\end{array}$ & $\begin{array}{c}F(\mathrm{H} \beta) \\
\left(10^{-13} \text { ergs }\right. \\
\left.\mathrm{s}^{-1} \mathrm{~cm}^{-2}\right) \\
(3)\end{array}$ \\
\hline $\begin{array}{l}8862 \ldots \ldots \ldots \\
8864 \ldots \ldots \ldots \\
8866 \ldots \ldots \ldots \\
8867 \ldots \ldots \ldots \\
8869 \ldots \ldots \ldots\end{array}$ & $\begin{array}{l}7.25 \pm 0.19 \\
7.53 \pm 0.30 \\
7.53 \pm 0.30 \\
7.50 \pm 0.22 \\
7.42 \pm 0.22\end{array}$ & $\begin{array}{l}6.07 \pm 0.22 \\
5.92 \pm 0.30 \\
5.98 \pm 0.30 \\
6.08 \pm 0.24 \\
6.14 \pm 0.25\end{array}$ & $\begin{array}{l}8876 \ldots \ldots \ldots \\
8883 \ldots \ldots \ldots \\
8886 \ldots \ldots \ldots \\
8889 \ldots \ldots \ldots \\
8898 \ldots \ldots \ldots\end{array}$ & $\begin{array}{l}7.87 \pm 0.24 \\
7.81 \pm 0.23 \\
7.83 \pm 0.23 \\
7.76 \pm 0.23 \\
7.03 \pm 0.21\end{array}$ & $\begin{array}{l}5.86 \pm 0.23 \\
5.97 \pm 0.24 \\
6.41 \pm 0.26 \\
6.47 \pm 0.26 \\
6.64 \pm 0.27\end{array}$ \\
\hline
\end{tabular}

$\tau-\Delta t / 2$ to $\tau+\Delta t / 2$; the correlation function is thus binned into discrete time elements of width of $\Delta t$. The modifications we have introduced into the original formulation of Edelson \& Krolik (1988) are (1) we do not weight the data points by their uncertainties, (2) we do not assume that the light curves are statistically stationary and we therefore normalize the correlation coefficient in each bin by using the mean and variance computed only from those data points in the bin, and (3) we do not adjust the variances by subtracting the uncertainties of the data points in quadrature (see Edelson \& Krolik, eq. [3]).

A cross-correlation analysis has been performed for each of the 4 separate years as well as for the data set in its entirety. The upper panels of Figure 4 show the interpolation CCFs and DCFs for each of the $4 \mathrm{yr}$. The lower panels show both the corresponding continuum autocorrelation functions (ACF) and the sampling window ACFs; the latter are produced by averaging the ACFs obtained by repeatedly sampling a whitenoise spectrum in exactly the same pattern as the real observations and then computing the ACF with the interpolation method. The interpolation process automatically introduces a correlation extending from zero lag to a value that characterizes the typical sampling interval, and thus the sampling window ACF provides a good indication of how much of the width of the ACF is introduced by the interpolation process rather than by real correlation of the continuum values at different times. The interpolation $\mathrm{CCF}, \mathrm{DCF}$, continuum ACF, and sampling wind'ow ACF for the entire $4 \mathrm{yr}$ experiment are shown in Figure 5. The characteristics of the CCFs are summarized in Table 7 . Here the parameter $r_{\max }$ is the peak value of the interpolation CCF, which occurs at a time delay $\Delta t_{\text {Peak }}$, and $\Delta t_{\text {Center }}$ refers to the median of the half-maximum points. The uncertainties in these values are typically $\sim 2-4$ days. As before, we caution against ascribing much physical significance to any single number that is used to characterize the CCF, as interpolation of both the lag and its uncertainty depends on numerous factors, including the geometry of the BLR and on the continuum behavior (cf. Robinson \& Pérez 1990). The relatively small uncertainty in the lag and the similarity of the solutions for the separate years shows that it is, however, a well-defined and experimentally repeatable quantity.

\subsection{The $\mathrm{H} \beta$ Transfer Function}

It is usually assumed that the relationship between the continuum light curve $C(t)$ and the emission-line light curve $L(t)$ can be written

$$
L(t)=\int_{-\infty}^{\infty} \Psi(\tau) C(t-\tau) d \tau,
$$

where $\Psi(\tau)$ is the geometry-dependent transfer function (Blandford \& McKee 1982). In principle, with the obervables $C(t)$ and $L(t)$, equation (3) can be inverted to solve for the transfer function. Transfer function solutions for NGC 5548, which have been obtained by employing a maximum entropy method (MEM), have been presented by Krolik et al. (1991) for the UV lines and by Horne, Welsh, \& Peterson (1991) for $\mathbf{H} \beta$. We have used the data described here and in Papers II and III to construct a MEM solution for the transfer function for

TABLE 6

VARIABILITy Parameters and SAMPling Characteristics

\begin{tabular}{|c|c|c|c|c|c|c|}
\hline \multirow[b]{2}{*}{$\begin{array}{l}\text { Feature } \\
\text { (1) }\end{array}$} & \multirow[b]{2}{*}{$\begin{array}{c}\text { NUMBER } \\
\text { OF EPOCHS } \\
\text { (2) }\end{array}$} & \multicolumn{2}{|c|}{$\begin{array}{c}\text { SAMPLING } \\
\text { INTERVAL (days) }\end{array}$} & \multirow[b]{2}{*}{$\begin{array}{c}\text { MEAN } \\
\text { FLUX } \\
\text { (5) }\end{array}$} & \multirow[b]{2}{*}{$\begin{array}{c}F_{\text {var }} \\
(6)\end{array}$} & \multirow[b]{2}{*}{$\begin{array}{r}R_{\mathrm{ma}} \\
\text { (7) }\end{array}$} \\
\hline & & $\begin{array}{l}\text { Average } \\
\text { (3) }\end{array}$ & $\begin{array}{c}\text { Median } \\
\text { (4) }\end{array}$ & & & \\
\hline \multicolumn{7}{|c|}{$F_{\lambda}(5100 \AA)$} \\
\hline 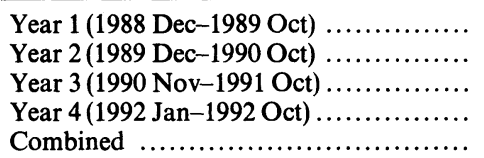 & $\begin{array}{r}125 \\
94 \\
65 \\
83 \\
367\end{array}$ & $\begin{array}{l}2.4 \\
3.4 \\
4.8 \\
3.4 \\
3.8\end{array}$ & $\begin{array}{l}1 \\
2 \\
3 \\
2 \\
2\end{array}$ & $\begin{array}{l}9.92 \\
7.25 \\
9.40 \\
6.72 \\
8.42\end{array}$ & $\begin{array}{l}0.123 \\
0.133 \\
0.094 \\
0.169 \\
0.210\end{array}$ & $\begin{array}{l}2.16 \\
1.83 \\
1.51 \\
2.04 \\
2.51\end{array}$ \\
\hline \multicolumn{7}{|c|}{$\mathrm{H} \beta$} \\
\hline 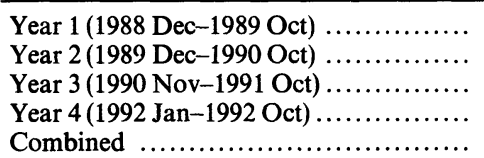 & $\begin{array}{r}132 \\
94 \\
65 \\
83 \\
374\end{array}$ & $\begin{array}{l}2.3 \\
3.4 \\
4.8 \\
3.4 \\
3.7\end{array}$ & $\begin{array}{l}1 \\
2 \\
3 \\
2 \\
2\end{array}$ & $\begin{array}{l}8.62 \\
5.98 \\
7.46 \\
4.96 \\
6.94\end{array}$ & $\begin{array}{l}0.095 \\
0.191 \\
0.102 \\
0.284 \\
0.260\end{array}$ & $\begin{array}{l}1.57 \\
2.30 \\
1.58 \\
3.03 \\
4.04\end{array}$ \\
\hline
\end{tabular}

a Units as in Table 5. 


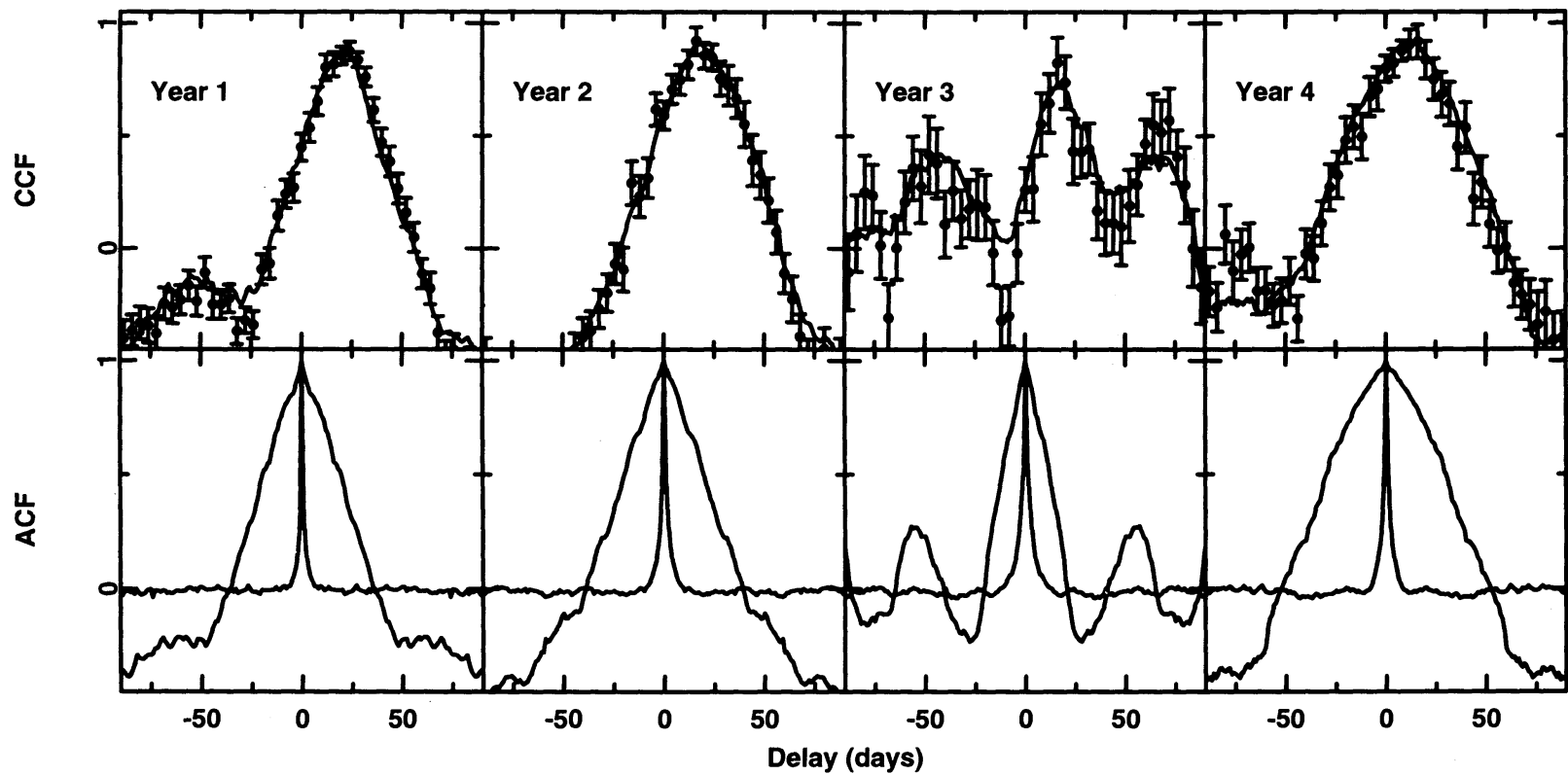

FIG. 4.- The cross-correlation functions for the optical continuum $(5100 \AA)$ and the $\mathrm{H} \beta$ emission line, for each of the $4 \mathrm{yr}$ of this monitoring proram, are shown in the top row of panels. The interpolation CCF is shown as a smooth line, and the DCF values are plotted as individual points with associated uncertainties. The bin width for the DCF is 4 days. The corresponding continuum autocorrelation functions appear in the bottom row. Also illustrated in the bottom panels are the sampling window autocorrelations functions, which show the effect of interpolating the data between observations (Gaskell \& Peterson 1987).

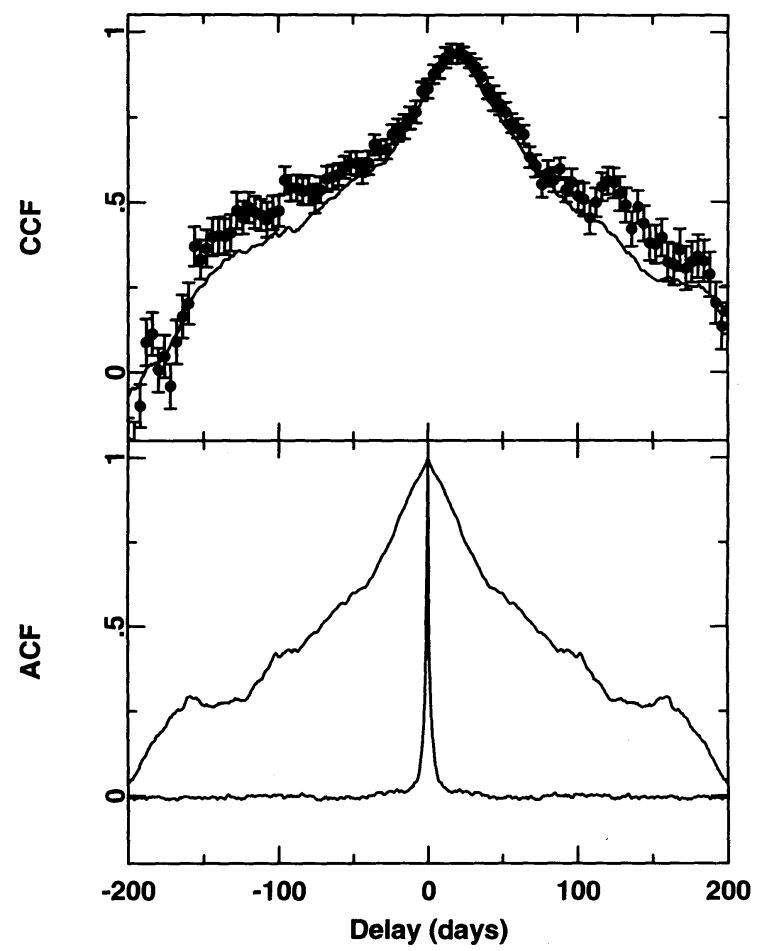

Fig. 5.-The top panel shows the optical continuum-H $\beta$ cross-correlation function for the entire $4 \mathrm{yr}$ monitoring program. The interpolation CCF is shown as a smooth line, and the DCF values are plotted as individual points with associated uncertainties. The bin width for the DCF is 4 days. The optical continuum autocorrelation function and the very narrow sampling window autocorrelation function appear in the lower panel. each of the $4 \mathrm{yr}$ separately and for the database as a whole. The details of the solution are discussed more completely by Peterson et al. (1993). The results of this analysis are shown in Figure 6. The transfer function for the total data set is quite similar to that derived from the first year's data alone and the expanded database appears to eliminate some of the structure at large lags that Horne et al. (1991) suspected was attributable to aliasing. However, the solutions for the separate years show some differences from year to year, particularly in year 4 (1992), when the peak response was more rapid than in previous years, as shown by the cross-correlation results (Table 7).

\section{CONCLUSIONS}

The optical spectroscopic monitoring program on the Seyfert 1 galaxy NGC 5548, described in Papers II and III, has been extended by 2 more years to provide a total baseline of nearly 1400 days. In this contribution, we have presented the first results from this extended campaign:

1. The $\mathrm{H} \beta$ emission-line variations follow those of the continuum on a timescale of about 18 days.

2. The continuum variations in NGC 5548 are usually rela-

TABLE 7

Cross-Correlation Results Optical ContinuUm vs. H $\beta$

\begin{tabular}{|c|c|c|c|}
\hline $\begin{array}{l}\text { Data Set } \\
\text { (1) }\end{array}$ & $\begin{array}{c}\Delta t_{\text {Peak }} \\
\text { (days) } \\
\text { (2) }\end{array}$ & $\begin{array}{c}\Delta t_{\text {Center }} \\
\text { (days) } \\
\text { (3) }\end{array}$ & $\begin{array}{c}r_{\max } \\
(4)\end{array}$ \\
\hline Year 1 (1988 Dec-1989 Oct) & 22 & 19.4 & 0.87 \\
\hline Year 2(1989 Dec-1990 Oct) & 18 & 19.4 & 0.91 \\
\hline Year 3 (1990 Dec-1991 Oct) ....... & 18 & 18.5 & 0.74 \\
\hline Year 4 (1992 Jan-1992 Oct) ... & 14 & 10.3 & 0.92 \\
\hline Combined $\ldots \ldots \ldots \ldots \ldots \ldots$ & 18 & 18.1 & 0.93 \\
\hline
\end{tabular}




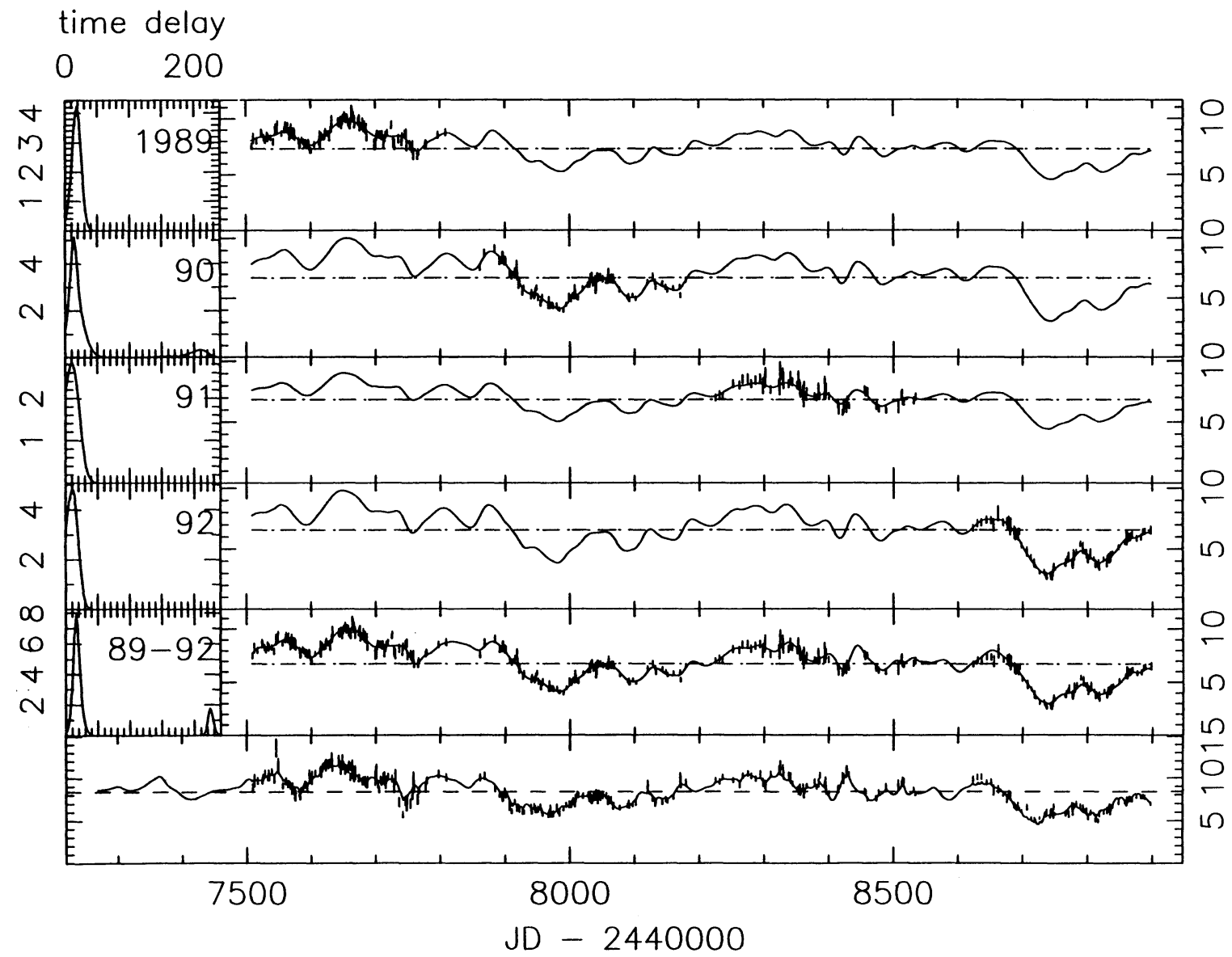

Fig. 6.-The $\mathrm{H} \beta$ transfer function for each of the $4 \mathrm{yr}$ and for the entire $4 \mathrm{yr}$ monitoring period. The bottom panel shows the maximum entropy fit to the observed continuum points, shown as vertical lines which correspond to $1 \sigma$ uncertainties. In the four upper panels, the transfer functions for the various subsets of the data (each individual year plus the entire data base) are shown on the left side and the right side of each panel shows the maximum entropy fit to the $\mathrm{H} \beta$ observations, also shown as $1 \sigma$ error bars. The dashed horizontal lines show the mean values of the light curves.

tively slow and well-resolved with the typical sampling of the experiment (2-4 days). However, during the third year the continuum variations were more rapid and of lower amplitude, similar to those observed in NGC 3783 in 1992, as described in Papers V and VI.

3. NGC 5548 went through an extremely faint state in 1992. The normally weak narrow components of the emission lines and stellar absorption features are relatively prominent in spectra obtained during this period and will be very useful in assessing how all of the data are affected by these nonvariable components.

4. The $\mathrm{H} \beta$ transfer function derived from the entire $4 \mathrm{yr}$ database is similar to that obtained by Horne et al. (1991) for the first year's data. The transfer function is narrow and peaked at around 18 days with very low amplitude at zero lag. However, the transfer functions for the 4 separate years show some differences, notably that the peak response during the fourth year (1992) is at smaller time delays than in the previous years.

We are very grateful to the Directors and Telescope Allocation Committees of our various observatories for their support of this project. Individual investigators have benefitted from support from a number of agencies, including the following: the National Science Foundation: AST-9117086 (Ohio State University), AST-8714937 (University of Texas), AST-8957063 and AST-9003829 (University of California, Berkeley), and the NSF Graduate Fellowship program (T. M.); NASA: NAG51824 (Ohio State University), NAS8-30751 (Center for Astrophysics), and Long-Term Space Astrophysics grants NAGW-2678 (K. H.) and NAGW-3315 (B. M. P.); and BMFT grant Verbundforschung Astronomie DFG Ko857/13-1 (Universitäts-Sternwarte Göttingen). We thank the referee, D. Maoz, for helpful comments.
Blandford, R. D., \& McKee, C. F. 1982, ApJ, 255, 419

Clavel, J., et al. 1991, ApJ, 366, 64 (Paper I)

Dietrich, M.. et al. 1993, ApJ, 408, 416 (Paper IV)

Edelson, R. A., \& Krolik, J. H. 1988, ApJ, 333, 646

Gaskell, C. M., \& Peterson, B. M. 1987, ApJS, 65, 1

Gaskell, C. M., \& Sparke, L. S. 1986, ApJ, 305, 175

Horne, K., Welsh, W.F., \& Peterson, B. M. 1991, ApJ, 367, L5

Iijima, T., Rafanelli, P., \& Bianchini, A. 1992, A\&A, 265, L25

Krolik, J. H., Horne, K., Kallman, T. R., Malkan, M. A., Edelson, R. A., \&

Kriss, G. A. 1991, ApJ, 371, 541

Maoz, D., et al. 1993, ApJ, 404, 576

\section{REFERENCES}

Peterson, B. M. 1993, PASP, 105, 247

Peterson, B. M., et al. 1991, ApJ, 368, 119 (Paper II)

- 1992, ApJ, 392, 470 (Paper III)

Peterson, B. M., Ali, B., Horne, K., Bertram, R., Lame, N. J., Pogge, R. W., \& Wagner, R. M. 1993, ApJ, 402, 469

Reichert, G. A., et al. 1994, ApJ, 425, 582 (Paper V)

Robinson, A., \& Pérez, E. 1990, MNRAS, 244, 138

Stirpe, G. M., et al. 1994, ApJ, 425, 609 (Paper VI)

Wanders, I., Peterson, B. M., Pogge, R. W., DeRobertis, M. M., \& van Groningen, E. 1992, A\&A, 266, 72 